\title{
Knowledge and Common Knowledge in a Distributed Environment*
}

\author{
Joseph Y. Halpern \\ IBM Almaden Research Center \\ San Jose, CA 95120
}

\author{
Yoram Moses
}

\begin{abstract}
Reasoning about knowledge seems to play a fundamental role in distributed systems. Indeed, such reasoning is a central part of the informal intuitive arguments used in the design of distributed protocols. Communication in a distributed system can be viewed as the act of transforming the system's state of knowledge. This paper presents a general framework for formalizing and reasoning about knowledge in distributed systems. We argue that states of knowledge of groups of processors are useful concepts for the design and analysis of distributed protocols. In particular, distributed knowledge corresponds to knowledge that is "distributed" among the members of the group, while common knowledge corresponds to a fact being "publicly known". The relationship between common knowledge and a variety of desirable actions in a distributed system is illustrated. Furthermore, it is shown that, formally speaking, in practical systems common knowledge cannot be attained. A number of weaker variants of common knowledge that are attainable in many cases of interest are introduced and investigated.
\end{abstract}

\footnotetext{
${ }^{*}$ This is a revised and expanded version of a paper with the same title that first appeared in the Proceedings of the 3rd ACM Conference on Principles of Distributed Computing, 1984. It is essentially identical to the version that appears in Journal of the ACM 37:3, 1990, pp. 549-587. The work of the second author was supported in part by DARPA contract N00039-82-C-0250.
} 


\section{Introduction}

Distributed systems of computers are rapidly gaining popularity in a wide variety of applications. However, the distributed nature of control and information in such systems makes the design and analysis of distributed protocols and plans a complex task. In fact, at the current time, these tasks are more an art than a science. Basic foundations, general techniques, and a clear methodology are needed to improve our understanding and ability to deal effectively with distributed systems.

While the tasks that distributed systems are required to perform are normally stated in terms of the global behavior of the system, the actions that a processor performs can depend only on its local information. Since the design of a distributed protocol involves determining the behavior and interaction between individual processors in the system, designers frequently find it useful to reason intuitively about processors' "states of knowledge" at various points in the execution of a protocol. For example, it is customary to argue that "... once the sender receives the acknowledgement, it knows that the current packet has been delivered; it can then safely discard the current packet, and send the next packet...". Ironically, however, formal descriptions of distributed protocols, as well as actual proofs of their correctness or impossibility, have traditionally avoided any explicit mention of knowledge. Rather, the intuitive arguments about the state of knowledge of components of the system are customarily buried in combinatorial proofs that are unintuitive and hard to follow.

The general concept of knowledge has received considerable attention in a variety of fields, ranging from Philosophy [Hin62 and Artificial Intelligence [MSHI79] and Moo85, to Game Theory Aum76 and Psychology CM81. The main purpose of this paper is to demonstrate the relevance of reasoning about knowledge to distributed systems as well. Our basic thesis is that explicitly reasoning about the states of knowledge of the components of a distributed system provides a more general and uniform setting that offers insight into the basic structure and limitations of protocols in a given system.

As mentioned above, agents can only base their actions on their local information. This knowledge, in turn, depends on the messages they receive and the events they observe. Thus, there is a close relationship between knowledge and action in a distributed environment. When we consider the task of performing coordinated actions among a number of agents in a distributed environment, it does not, in general, suffice to talk only about individual agents' knowledge. Rather, we need to look at states of knowledge of groups of agents (the group of all participating agents is often the most relevant one to consider). Attaining particular states of group knowledge is a prerequisite for performing coordinated actions of various kinds.

In this work we define a hierarchy of states of group knowledge. It is natural to think of communication in the system as the act of improving the state of knowledge, in the sense of "climbing up the hierarchy". The weakest state of knowledge we discuss is distributed knowledge, which corresponds to knowledge that is distributed among the 
members of the group, without any individual agent necessarily having it. The strongest state of knowledge in the hierarchy is common knowledge, which roughly corresponds to "public knowledge". We show that the execution of simultaneous actions becomes common knowledge, and hence that such actions cannot be performed if common knowledge cannot be attained. Reaching agreement is an important example of a desirable simultaneous action in a distributed environment. A large part of the technical analysis in this paper is concerned with the ability and cost of attaining common knowledge in systems of various types. It turns out that attaining common knowledge in distributed environments is not a simple task. We show that when communication is not guaranteed it is impossible to attain common knowledge. This generalizes the impossibility of a solution to the well-known coordinated attack problem Gra78. A more careful analysis shows that common knowledge can only be attained in systems that support simultaneous coordinated actions. It can be shown that such actions cannot be guaranteed or detected in practical distributed systems. It follows that common knowledge cannot be attained in many cases of interest. We then consider states of knowledge that correspond to eventually coordinated actions and to coordinated actions that are guaranteed to be performed within a bounded amount of time. These are essentially weaker variants of common knowledge. However, whereas, strictly speaking, common knowledge may be difficult to attain in many practical cases, these weaker states of knowledge are attainable in cases of interest.

Another question that we consider is that of when it is safe to assume that certain facts are common knowledge, even when strictly speaking they are not. For this purpose, we introduce the concept of internal knowledge consistency. Roughly speaking, it is internally knowledge consistent to assume that a certain state of knowledge holds at a given point, if nothing the processors in the system will ever encounter will be inconsistent with this assumption.

The rest of the paper is organized as follows. In the next section we look at the "muddy children" puzzle, which illustrates some of the subtleties involved in reasoning about knowledge in the context of a group of agents. In Section 3 we introduce a hierarchy of states of knowledge in which a group may be. Section 4 focuses on the relationship between knowledge and communication by looking at the coordinated attack problem. In Section 5 we sketch a general definition of a distributed system, and in Section 6 we discuss how knowledge can be ascribed to processors in such systems so as to make statements such as "agent 1 knows $\varphi$ " completely formal and precise. Section 7 relates common knowledge to the coordinated attack problem. In Section 8, we show that, strictly speaking, common knowledge cannot be attained in practical distributed systems. Section 9 considers the implications of this observation and in Section 10 we begin to reconsider the notion of common knowledge in the light of these implications. In Sections 11 and 12, we consider a number of variants of common knowledge that are

\footnotetext{
${ }^{1}$ In a previous version of this paper [HM90], what we are now calling distributed knowledge was called implicit knowledge. We have changed the name here to avoid conflict with the usage of the phrase "implicit knowledge" in papers such as [FH88, Lev84].
} 
attainable in many cases of interest and discuss the relevance of these states of knowledge to the actions that can be performed in a distributed system. Section 13 discusses the notion of internal knowledge consistency, and Section 14 contains some concluding remarks.

\section{The muddy children puzzle}

A crucial aspect of distributed protocols is the fact that a number of different processors cooperate in order to achieve a particular goal. In such cases, since more than one agent is present, an agent may have knowledge about other agents' knowledge in addition to his knowledge about the physical world. This often requires care in distinguishing subtle differences between seemingly similar states of knowledge. A classical example of this phenomenon is the muddy children puzzle - a variant of the well known "wise men" or "cheating wives" puzzles. The version given here is taken from [Bar81]:

Imagine $n$ children playing together. The mother of these children has told them that if they get dirty there will be severe consequences. So, of course, each child wants to keep clean, but each would love to see the others get dirty. Now it happens during their play that some of the children, say $k$ of them, get mud on their foreheads. Each can see the mud on others but not on his own forehead. So, of course, no one says a thing. Along comes the father, who says, "At least one of you has mud on your head," thus expressing a fact known to each of them before he spoke (if $k>1$ ). The father then asks the following question, over and over: "Can any of you prove you have mud on your head?" Assuming that all the children are perceptive, intelligent, truthful, and that they answer simultaneously, what will happen?

The reader may want to think about the situation before reading the rest of Barwise's discussion:

There is a "proof" that the first $k-1$ times he asks the question, they will all say "no" but then the $k$ th time the dirty children will answer "yes."

The "proof" is by induction on $k$. For $k=1$ the result is obvious: the dirty child sees that no one else is muddy, so he must be the muddy one. Let us do $k=2$. So there are just two dirty children, $a$ and $b$. Each answers "no" the first time, because of the mud on the other. But, when $b$ says "no," $a$ realizes that he must be muddy, for otherwise $b$ would have known the mud was on his head and answered "yes" the first time. Thus $a$ answers "yes" the second time. But $b$ goes through the same reasoning. Now suppose $k=3$; so there are three dirty children, $a, b, c$. Child $a$ argues as follows. Assume I don't have mud on my head. Then, by the $k=2$ case, both $b$ and $c$ will answer 
"yes" the second time. When they don't, he realizes that the assumption was false, that he is muddy, and so will answer "yes" on the third question. Similarly for $b$ and $c$. [The general case is similar.]

Let us denote the fact "At least one child has a muddy forehead" by $\mathbf{m}$. Notice that if $k>1$, i.e., more than one child has a muddy forehead, then every child can see at least one muddy forehead, and the children initially all know $\mathbf{m}$. Thus, it would seem, the father does not need to tell the children that $\mathbf{m}$ holds when $k>1$. But this is false! In fact, had the father not announced $\mathbf{m}$, the muddy children would never have been able to conclude that their foreheads are muddy. We now sketch a proof of this fact.

First of all, given that the children are intelligent and truthful, a child with a clean forehead will never answer "yes" to any of the father's questions. Thus, if $k=0$, all of the children answer all of the father's questions "no". Assume inductively that if there are exactly $k$ muddy children and the father does not announce $\mathbf{m}$, then the children all answer "no" to all of the father's questions. Note that, in particular, when there are exactly $k$ muddy foreheads, a child with a clean forehead initially sees $k$ muddy foreheads and hears all of the father's questions answered "no". Now assume that there are exactly $k+1$ muddy children. Let $q \geq 1$ and assume that all of the children answer "no" to the father's first $q-1$ questions. We have argued above that a clean child will necessarily answer "no" to the father's $q^{\text {th }}$ question. Next observe that before answering the father's

$q^{\text {th }}$ question, a muddy child has exactly the same information as a clean child has at the corresponding point in the case of $k$ muddy foreheads. It follows that the muddy children must all answer "no" to the father's $q^{\text {th }}$ question, and we are done. (A very similar proof shows that if there are $k$ muddy children and the father does announce $\mathbf{m}$, his first $k-1$ questions are answered "no".)

So, by announcing something that the children all know, the father somehow manages to give the children useful information! How can this be? Exactly what was the role of the father's statement? In order to answer this question, we need to take a closer look at knowledge in the presence of more than one knower; this is the subject of the next section.

\section{A hierarchy of states of knowledge}

In order to analyze the muddy children puzzle introduced in the previous section, we need to consider states of knowledge of groups of agents. As we shall see in the sequel, reasoning about such states of knowledge is crucial in the context of distributed systems as well. In Section 6 we shall carefully define what it means for an agent $i$ to know a given fact $\varphi$ (which we denote by $K_{i} \varphi$ ). For now, however, we need knowledge to satisfy only two properties. The first is that an agent's knowledge at a given time must depend only on its local history: the information that it started out with combined with the events it has observed since then. Secondly, we require that only true things be known, 
or more formally:

$$
K_{i} \varphi \supset \varphi ;
$$

i.e., if an agent $i$ knows $\varphi$, then $\varphi$ is true. This property, which is occasionally referred to as the knowledge axiom, is the main property that philosophers customarily use to distinguish knowledge from belief (cf. [HM92]).

Given a reasonable interpretation for what it means for an agent to know a fact $\varphi$, how does the notion of knowledge generalize from an agent to a group? In other words, what does it mean to say that a group $G$ of agents knows a fact $\varphi$ ? We believe that more than one possibility is reasonable, with the appropriate choice depending on the application:

- $D_{G} \varphi$ (read "the group $G$ has distributed knowledge of $\varphi$ "): We say that knowledge of $\varphi$ is distributed in $G$ if someone who knew everything that each member of $G$ knows would know $\varphi$. For instance, if one member of $G$ knows $\psi$ and another knows that $\psi \supset \varphi$, the group $G$ may be said to have distributed knowledge of $\varphi$.

- $S_{G} \varphi$ (read "someone in $G$ knows $\varphi$ "): We say that $S_{G} \varphi$ holds iff some member of $G$ knows $\varphi$. More formally,

$$
S_{G} \varphi \equiv \bigvee_{i \in G} K_{i} \varphi
$$

- $E_{G} \varphi$ (read "everyone in $G$ knows $\varphi$ "): We say that $E_{G} \varphi$ holds iff all members of $G$ know $\varphi$. More formally,

$$
E_{G} \varphi \equiv \bigwedge_{i \in G} K_{i} \varphi .
$$

- $E_{G}^{k} \varphi$, for $k \geq 1 \quad\left(\right.$ read " $\varphi$ is $E^{k}$-knowledge in $G$ "): $E_{G}^{k} \varphi$ is defined by

$$
\begin{gathered}
E_{G}^{1} \varphi=E_{G} \varphi, \\
E_{G}^{k+1} \varphi=E_{G} E_{G}^{k} \varphi, \text { for } k \geq 1 .
\end{gathered}
$$

$\varphi$ is said to be $E^{k}$-knowledge in $G$ if "everyone in $G$ knows that everyone in $G$ knows that ... that everyone in $G$ knows that $\varphi$ is true" holds, where the phrase "everyone in $G$ knows that" appears in the sentence $k$ times.

- $C_{G} \varphi$ (read " $\varphi$ is common knowledge in $G$ "): The formula $\varphi$ is said to be common knowledge in $G$ if $\varphi$ is $E_{G}^{k}$-knowledge for all $k \geq 1$. In other words,

$$
C_{G} \varphi \equiv E_{G} \varphi \wedge E_{G}^{2} \varphi \wedge \cdots \wedge E_{G}^{m} \varphi \wedge \cdots
$$

(We omit the subscript $G$ when the group $G$ is understood from context.) 
Clearly, the notions of group knowledge introduced above form a hierarchy, with

$$
C \varphi \supset \cdots \supset E^{k+1} \varphi \supset \cdots \supset E \varphi \supset S \varphi \supset D \varphi \supset \varphi .
$$

However, depending on the circumstances, these notions might not be distinct. For example, consider a model of parallel computation in which a collection of $n$ processors share a common memory. If their knowledge is based on the contents of the common memory, then we arrive at a situation in which $C \varphi \equiv E^{k} \varphi \equiv E \varphi \equiv S \varphi \equiv D \varphi$. By way of contrast, in a distributed system in which $n$ processors are connected via some communication network and each one of them has its own memory, the above hierarchy is strict. Moreover, in such a system, every two levels in the hierarchy can be separated by an actual task, in the sense that there will be an action for which one level in the hierarchy will suffice, but no lower level will. It is quite clear that this is the case with $E \varphi \supset S \varphi \supset D \varphi$, and, as we are about to show, the "muddy children" puzzle is an example of a situation in which $E^{k} \varphi$ suffices to perform a required action, but $E^{k-1} \varphi$ does not. In the next section we present the coordinated attack problem, a problem for which $C \varphi$ suffices to perform a required action, but for no $k$ does $E^{k} \varphi$ suffice.

Returning to the muddy children puzzle, let us consider the state of the children's knowledge of $\mathbf{m}$ : "At least one forehead is muddy". Before the father speaks, $E^{k-1} \mathbf{m}$ holds, and $E^{k} \mathbf{m}$ doesn't. To see this, consider the case $k=2$ and suppose that Alice and Bob are the only muddy children. Clearly everyone sees at least one muddy child, so Em holds. But the only muddy child that Alice sees is Bob, and, not knowing whether she is muddy, Alice considers it possible that Bob is the only muddy child. Alice therefore considers it possible that Bob sees no muddy child. Thus, although both Alice and Bob know $\mathbf{m}$ (i.e., Em holds), Alice does not know that Bob knows $\mathbf{m}$, and hence $E^{2} \mathbf{m}$ does not hold. A similar argument works for the general case. We leave it to the reader to check that when there are $k$ muddy children, $E^{k} \mathbf{m}$ suffices to ensure that the muddy children will be able to prove their dirtiness, whereas $E^{k-1} \mathbf{m}$ does not. (For a more detailed analysis of this argument, and for a general treatment of variants of the muddy children puzzle, see MDH86.)

Thus, the role of the father's statement was to improve the children's state of knowledge of $\mathbf{m}$ from $E^{k-1} \mathbf{m}$ to $E^{k} \mathbf{m}$. In fact, the children have common knowledge of $\mathbf{m}$ after the father announces that $\mathbf{m}$ holds. Roughly speaking, the father's public announcement of $\mathbf{m}$ to the children as a group results in all the children knowing $\mathbf{m}$ and knowing that the father has publicly announced $\mathbf{m}$. Assuming that it is common knowledge that all of the children know anything the father announces publicly, it is easy to conclude that the father's announcement makes $\mathbf{m}$ common knowledge. Once the father announces $\mathbf{m}$, all of the children know both $\mathbf{m}$ and that the father has announced $\mathbf{m}$. Every child thus knows that all of the children know $\mathbf{m}$ and know that the father publicly announced $\mathbf{m}$, and so $E^{2} \mathbf{m}$ holds. It is similarly possible to show that once the father announces $\mathbf{m}$ then $E^{k} \mathbf{m}$ holds for all $k$, so $C \mathbf{m}$ holds (see Section 10 for further discussion). Since, in particular, $E^{k} \mathbf{m}$ holds, the muddy children can succeed in proving their dirtiness. 
The vast majority of the communication in a distributed system can also be viewed as the act of improving the state of knowledge (in the sense of "climbing up a hierarchy") of certain facts. This is an elaboration of the view of communication in a network as the act of "sharing knowledge". Taking this view, two notions come to mind. One is fact discovery - the act of changing the state of knowledge of a fact $\varphi$ from being distributed knowledge to levels of explicit knowledge (usually $S$-, $E$-, or $C$-knowledge), and the other is fact publication - the act of changing the state of knowledge of a fact that is not common knowledge to common knowledge. An example of fact discovery is the detection of global properties of a system, such as deadlock. The system initially has distributed knowledge of the deadlock, and the detection algorithm improves this state to $S$-knowledge (see CL85 for work related to fact discovery). An example of fact publication is the introduction of a new communication convention in a computer network. Here the initiator(s) of the convention wish to make the new convention common knowledge.

In the rest of the paper we devote a considerable amount of attention to fact publication and common knowledge. As we shall show, common knowledge is inherent in a variety of notions of agreement, conventions, and coordinated action. Furthermore, having common knowledge of a large number of facts allows for more efficient communication. Since these are goals frequently sought in distributed computing, the problem of fact publication - how to attain common knowledge - becomes crucial. Common knowledge is also a basic notion in everyday communication between people. For example, shaking hands to seal an agreement signifies that the handshakers have common knowledge of the agreement. Also, it can be argued [CM81 that when we use a definite reference such as "the president" in a sentence, we assume common knowledge of who is being referred to.

In [CM81, Clark and Marshall present two basic ways in which a group can come to have common knowledge of a fact. One is by membership in a community, e.g., the meaning of a red traffic light is common knowledge in the community of licensed drivers. The other is by being copresent with the occurrence of the fact, e.g., the father's gathering the children and publicly announcing the existence of muddy foreheads made that fact common knowledge. Notice that if, instead, the father had taken each child aside (without the other children noticing) and told her or him about it privately, this information would have been of no help at all.

In the context of distributed systems, community membership corresponds to information that the processors are guaranteed to have by virtue of their presence in the system (e.g., information that is "inserted into" the processors before they enter the system). However, it is not obvious how to simulate copresence or "public" announcements using message passing in a distributed system. As we shall see, there are serious problems and unexpected subtleties involved in attempting to do so. 


\section{The coordinated attack problem}

To get a flavor of the issues involved in attaining common knowledge by simulating copresence in a distributed system, consider the coordinated attack problem, originally introduced by Gray Gra78:

Two divisions of an army are camped on two hilltops overlooking a common valley. In the valley awaits the enemy. It is clear that if both divisions attack the enemy simultaneously they will win the battle, whereas if only one division attacks it will be defeated. The divisions do not initially have plans for launching an attack on the enemy, and the commanding general of the first division wishes to coordinate a simultaneous attack (at some time the next day). Neither general will decide to attack unless he is sure that the other will attack with him. The generals can only communicate by means of a messenger. Normally, it takes the messenger one hour to get from one encampment to the other. However, it is possible that he will get lost in the dark or, worse yet, be captured by the enemy. Fortunately, on this particular night, everything goes smoothly. How long will it take them to coordinate an attack?

We now show that despite the fact that everything goes smoothly, no agreement can be reached and no general can decide to attack. (This is, in a way, a folk theorem of operating systems theory; cf. [Gal79, Gra78, YC79].) Suppose General $A$ sends a message to General $B$ saying "Let's attack at dawn", and the messenger delivers it an hour later. General $A$ does not immediately know whether the messenger succeeded in delivering the message. And because $B$ would not attack at dawn if the messenger is captured and fails to deliver the message, $A$ will not attack unless he knows that the message was successfully delivered. Consequently, $B$ sends the messenger back to $A$ with an acknowledgement. Suppose the messenger delivers the acknowledgement to $A$ an hour later. Since $B$ knows that $A$ will not attack without knowing that $B$ received the original message, he knows that $A$ will not attack unless the acknowledgement is successfully delivered. Thus, $B$ will not attack unless he knows that the acknowledgement has been successfully delivered. However, for $B$ to know that the acknowledgement has been successfully delivered, $A$ must send the messenger back with an acknowledgement to the acknowledgement .... Similar arguments can be used to show that no fixed finite number of acknowledgements, acknowledgements to acknowledgements, etc. suffices for the generals to attack. Note that in the discussion above the generals are essentially running a handshake protocol (cf. Gra78). The above discussion shows that for no $k$ does a $k$-round handshake protocol guarantee that the generals be able to coordinate an attack.

In fact, we can use this intuition to actually prove that the generals can never attack and be guaranteed that they are attacking simultaneously. We argue by induction on $d$

- the number of messages delivered by the time of the attack - that $d$ messages do not 
suffice. Clearly, if no message is delivered, then $B$ will not know of the intended attack, and a simultaneous attack is impossible. For the inductive step, assume that $k$ messages do not suffice. If $k+1$ messages suffice, then the sender of the $(k+1)^{\text {st }}$ message attacks without knowing whether his last message arrived. Since whenever one general attacks they both do, the intended receiver of the $(k+1)^{\text {st }}$ message must attack regardless of whether the $(k+1)^{\text {st }}$ message is delivered. Thus, the $(k+1)^{\text {st }}$ message is irrelevant, and $k$ messages suffice, contradicting the inductive hypothesis.

After presenting a detailed proof of the fact that no protocol the generals can use will satisfy their requirements and allow them to coordinate an attack, Yemini and Cohen in YC79 make the following remark:

... Furthermore, proving protocols correct (or impossible) is a difficult and cumbersome art in the absence of proper formal tools to reason about protocols. Such backward-induction argument as the one used in the impossibility proof should require less space and become more convincing with a proper set of tools.

Yemini and Cohen's proof does not explicitly use reasoning about knowledge, but it uses a many-scenarios argument to show that if the generals both attack in one scenario, then there is another scenario in which one general will attack and the other will not. The crucial point is that the actions that should be taken depend not only on the actual state of affairs (in this case, the messenger successfully delivering the messages), but also (and in an acute way) on what other states of affairs the generals consider possible. Knowledge is just the dual of possibility, so reasoning about knowledge precisely captures the manyscenario argument in an intuitive way. We feel that understanding the role knowledge plays in problems such as coordinated attack is a first step towards simplifying the task of designing and proving the correctness of protocols.

A protocol for the coordinated attack problem, if one did exist, would ensure that when the generals attack, they are guaranteed to be attacking simultaneously. Thus, in a sense, an attacking general (say $A$ ) would know that the other general (say $B$ ) is also attacking. Furthermore, $A$ would know that $B$ similarly knows that $A$ is attacking. It is easy to extend this reasoning to show that when the generals attack they have common knowledge of the attack. However, each message that the messenger delivers can add at most one level of knowledge about the desired attack, and no more. For example, when the message is first delivered to $B, B$ knows about $A$ 's desire to coordinate an attack, but $A$ does not know whether the message was delivered, and therefore $A$ does not know that $B$ knows about the intended attack. And when the messenger returns to $A$ with $B$ 's acknowledgement, $A$ knows that $B$ knows about the intended attack, but, not knowing whether the messenger delivered the acknowledgement, $B$ does not know that $A$ knows (that $B$ knows of the intended attack). This in some sense explains why the generals cannot reach an agreement to attack using a finite number of messages. We are about to formalize this intuition. Indeed, we shall prove a more general result from which the 
inability to achieve a guaranteed coordinated attack will follow as a corollary. Namely, we prove that communication cannot be used to attain common knowledge in a system in which communication is not guaranteed, and formally relate a guaranteed coordinated attack to attaining common knowledge. Before we do so, we need to define some of the terms that we use more precisely.

\section{$5 \quad$ A general model of a distributed system}

We now present a general model of a distributed environment. Formally, we model such an environment by a distributed system, where the agents are taken to be processors and interaction between agents is modeled by messages sent between the processors over communication links. For the sake of generality and applicability to problems involving synchronization in distributed systems, our treatment will allow processors to have hardware clocks. Readers not interested in such issues can safely ignore all reference to clocks made throughout the paper.

We view a distributed system as a finite collection $\left\{p_{1}, p_{2}, \ldots, p_{n}\right\}$ of two or more processors that are connected by a communication network. We assume an external source of "real time" that in general is not directly observable by the processors. The processors are state machines that possibly have clocks, where a clock is a monotone nondecreasing function of real time. If a processor has a clock, then we assume that its clock reading is part of its state. (This is in contrast to the approach taken by Neiger and Toueg in NT93; the difference is purely a matter of taste.) The processors communicate with each other by sending messages along the links in the network.

A run $r$ of a distributed system is a description of an execution of the system, from time 0 until the end of the execution. (We assume for simplicity that the system executes forever. If it terminates after finite time, we can just assume that it remains in the same state from then on.) A point is a pair $(r, t)$ consisting of a run $r$ and a time $t \geq 0$. We characterize the run $r$ by associating with each point $(r, t)$ every processor $p_{i}$ 's local history at $(r, t)$, denoted $h\left(p_{i}, r, t\right)$. Roughly speaking, $h\left(p_{i}, r, t\right)$ consists of the sequence of events that $p_{i}$ has observed up to time $t$ in run $r$. We now formalize this notion. We assume that processor $p_{i}$ "wakes up" or joins the system in run $r$ at some time $t_{\text {init }}\left(p_{i}, r\right) \geq 0$. The processor's local state when it wakes up is called its initial state. The initial configuration of a run consists of the initial state and the wake up time for each processor. In systems with clocks, the clock time function $\tau$ describes processors' clock readings; $\tau\left(p_{i}, r, t\right)$ is the reading of $p_{i}$ 's clock at the point $(r, t)$. Thus, $\tau\left(p_{i}, r, t\right)$ is undefined for $t<t_{\text {init }}\left(p_{i}, r\right)$ and is a monotonic nondecreasing function of $t$ for $t \geq t_{\text {init }}\left(p_{i}, r\right)$. We say that $r$ and $r^{\prime}$ have the same clock readings if $\tau\left(p_{i}, r, t\right)=\tau\left(p_{i}, r^{\prime}, t\right)$ for all processors $p_{i}$ and all times $t$. (If there are no clocks in the system, we say for simplicity that all runs have the same clock readings.) We take $h\left(p_{i}, r, t\right)$ to be empty if $t<t_{\text {init }}\left(p_{i}, r\right)$. For $t \geq t_{\text {init }}\left(p_{i}, r\right)$, the history $h\left(p_{i}, r, t\right)$ consists of $p_{i}$ 's initial state and the sequence of messages $p_{i}$ has sent and received up to, but not including, those sent or received at time $t$ (in the order they were 
sent/received). We assume that this sequence of messages is finite. If $p_{i}$ has a clock, the messages are also marked with the time at which they were sent or received (i.e., with $\tau\left(p_{i}, r, t\right)$, if they were sent or received at time $t$ ), and the history includes the range of values that the clock has read up to and including time $t$. If we consider randomized protocols, then $h\left(p_{i}, r, t\right)$ also includes $p_{i}$ 's random coin tosses. For ease of exposition, we restrict attention to deterministic protocols in this paper. In a deterministic system with no external inputs and no failures, a processor's internal state will be a function of its history. Thus, the sequence of internal states that a processor goes through can be recovered from its history.

Corresponding to every distributed system, given an appropriate set of assumptions about the properties of the system and its possible interaction with its environment, there is a natural set $R$ of all possible runs of the system. We identify a distributed system with such a set $R$ of its possible runs. For ease of exposition, we sometimes slightly abuse the language and talk about a point $(r, t)$ as being a point of $R$ when $r \in R$. A run $r^{\prime}$ is said to extend a point $(r, t)$ if $h\left(p_{i}, r, t^{\prime}\right)=h\left(p_{i}, r^{\prime}, t^{\prime}\right)$ for all $t^{\prime} \leq t$ and all processors $p_{i}$. Observe that $r^{\prime}$ extends $(r, t)$ iff $r$ extends $\left(r^{\prime}, t\right)$.

Identifying a system with a set of runs is an important idea that will play a crucial role in allowing us to make precise the meaning of knowledge in a distributed system. The relative behavior of clocks, the properties of communication in the system, and many other properties of the system, are directly reflected in the properties of this set of runs. Thus, for example, a system is synchronous exactly if in all possible runs of the system the processors and the communication medium work in synchronous phases. A truly asynchronous system is one in which the set of runs allows any message sent to be delayed an unbounded amount of time before being delivered. (We discuss asynchrony in greater detail in Section 8.) Clocks are guaranteed to be synchronized to within a bound of $\delta$ if they differ by no more than $\delta$ time units at all points in all runs of the system. If we view the set of runs as a probability space with some appropriate measure, then we can also capture probabilistic properties of the environment and formalize probabilistic protocols in this framework.

We shall often be interested in the set of runs generated by running a particular protocol, under some assumptions on the communication medium. Intuitively, a protocol is a function specifying what actions a processor takes (which in our case amounts to what messages it sends) at any given point (after the processor wakes up) as a function of the processor's local state. Since a processor's local state is determined by its history, we simply define a protocol to be a deterministic function specifying what messages the processor should send at any given instant, as a function of the processor's history. Recall that $h\left(p_{i}, r, t\right)$, processor $p_{i}$ 's history at the point $(r, t)$, does not include messages sent or received at time $t$, so a processor's actions at time $t$ according to a protocol depend only on messages received in the past. As we mentioned above, for ease of exposition we restrict attention to deterministic protocols in this paper. The definitions and results can be extended to nondeterministic and probabilistic protocols in a straightforward way. A joint protocol for $G$ is a tuple consisting of a protocol for every processor in $G$. 


\section{$6 \quad$ Ascribing knowledge to processors}

What does it mean to say that a processor knows a fact $\varphi$ ? In our opinion, there is no unique "correct" answer to this question. Different interpretations of knowledge in a distributed system are appropriate for different applications. For example, an interpretation by which a processor is said to know $\varphi$ only if $\varphi$ appears explicitly in a designated part of the processor's storage (its "database") seems interesting for certain applications. In other contexts we may be interested in saying that a processor knows $\varphi$ if the processor could deduce $\varphi$ from the information available to it. In this section we give precise definitions of interpretations of knowledge in a distributed system.

We assume the existence of an underlying logical language of formulas for representing ground facts about the system. A ground fact is a fact about the state of the system that does not explicitly involve processors' knowledge. For example, "the value of register $x$ is 0", or "processor $p_{i}$ sent the message $m$ to processor $p_{j}$ ", are ground facts.

We extend the original language of ground formulas to a language that is closed under operators for knowledge, distributed knowledge, everyone knows, and common knowledge (so that for every formula $\varphi$, processor $p_{i}$, and subset $G$ of the processors, $K_{i} \varphi, D_{G} \varphi$, $E_{G} \varphi$, and $C_{G} \varphi$ are formulas), and under Boolean connectives. (In Section 11 we consider additional operators.)

We now describe one of the most natural ways of ascribing knowledge to processors in a distributed system, which we call view-based knowledge interpretations. At every point each processor is assigned a view; we say that two points are indistinguishable to the processor if it has the same view in both. A processor is then said to know a fact at a given point exactly if the fact holds at all of the points that the processor cannot distinguish from the given one. Roughly speaking, a processor knows all of the facts that (information theoretically) follow from its view at the current point.]

More formally, a view function $v$ for a system $R$ assigns to every processor at any given point of $R$ a view from some set $\Sigma$ of views (the structure of $\Sigma$ is not relevant at this point); i.e., $v\left(p_{i}, r, t\right) \in \Sigma$ for each processor $p_{i}$ and point $(r, t)$ of $R$. Given that a processor's history captures all of the events in the system that a processor may possibly observe, we require the processor's view at any given point to be a function of its history at that point. In other words, whenever $h\left(p_{i}, r, t\right)=h\left(p_{i}, r^{\prime}, t^{\prime}\right)$, it must also be the case that $v\left(p_{i}, r, t\right)=v\left(p_{i}, r^{\prime}, t^{\prime}\right)$.

\footnotetext{
${ }^{2}$ In a previous version of this paper [HM90], view-based knowledge interpretations were called statebased interpretations. Particular view-based knowledge interpretations were first suggested to us independently by Cynthia Dwork and by Stan Rosenschein. Since the appearance of [HM90], most authors who considered knowledge in distributed systems have focussed on view-based interpretations; cf. CM86, DM90, FI86, HF85, LR86, MT88, PR85, RK86 and Hal87] for an overview. (See [FH88, Mos88 for examples of interpretations of knowledge that are not view based.) The approach taken to defining knowledge in view-based systems is closely related to the possible-worlds approach taken by Hintikka Hin62. For us the "possible worlds" are the points in the system; the "agents" are the processors. A processor in one world (i.e., point) considers another world possible if it has the same view in both.
} 
A view-based knowledge interpretation $\mathcal{I}$ is a triple $(R, \pi, v)$, consisting of a set of runs $R$, an assignment $\pi$ which associates with every point in $R$ a truth assignment to the ground facts (so that for every point $(r, t)$ in $R$ and every ground fact $P$, we have $\pi(r, t)(P) \in\{$ true, false $\}$ ), and a view function $v$ for $R$. A triple $(\mathcal{I}, r, t)$, where $\mathcal{I}$ is a knowledge interpretation and $(r, t)$ is a point of $R$, is called a knowledge point. Formulas are said to be true or false of knowledge points. Let $\mathcal{I}=(R, \pi, v)$. We can now define the truth of a formula $\varphi$ at a knowledge point $(\mathcal{I}, r, t)$, denoted $(\mathcal{I}, r, t) \models \varphi$ (and also occasionally read " $\varphi$ holds at $(\mathcal{I}, r, t)$ ", or just " $\varphi$ holds at $(r, t)$ ", if the interpretation $\mathcal{I}$ is clear from context), by induction on the structure of formulas:

(a) If $P$ is a ground formula then $(\mathcal{I}, r, t) \models P$ iff $\pi(r, t)(P)=$ true.

(b) $(\mathcal{I}, r, t) \models \neg \psi$ iff $(\mathcal{I}, r, t) \forall \psi$.

(c) $(\mathcal{I}, r, t) \models \psi_{1} \wedge \psi_{2}$ iff $(\mathcal{I}, r, t) \models \psi_{1}$ and $(\mathcal{I}, r, t) \models \psi_{2}$.

(d) $(\mathcal{I}, r, t) \models K_{i} \psi$ iff $\left(\mathcal{I}, r^{\prime}, t^{\prime}\right) \models \psi$ for all $\left(r^{\prime}, t^{\prime}\right)$ in $R$ satisfying $v\left(p_{i}, r, t\right)=v\left(p_{i}, r^{\prime}, t^{\prime}\right)$.

Part (a) says the truth value of ground facts is defined by $\pi$. Parts (b) and (c) state that negations and conjunctions have their classical meaning. Part (d) captures the fact a processor $p_{i}$ 's knowledge at a point $(r, t)$ is completely determined by its view $v\left(p_{i}, r, t\right)$. The processor does not know $\varphi$ in a given view exactly if there is a point (in $R$ ) at which the processor has that same view, and $\varphi$ does not hold. The definitions of when $E_{G} \varphi$ and $C_{G} \varphi$ hold at a knowledge point follow directly from the definition of $E_{G}$ and $C_{G}$ in Section 3:

(e) $(\mathcal{I}, r, t) \models E_{G} \psi$ iff $(\mathcal{I}, r, t) \models K_{i} \psi$ for all $p_{i} \in G$.

(f) $(\mathcal{I}, r, t) \models C_{G} \psi$ iff $(\mathcal{I}, r, t) \models E_{G}^{k} \psi$ for all $k>0$.

Let us consider when a group $G$ of processors has distributed knowledge of a fact. Intuitively, a group's distributed knowledge is the combined knowledge of all of its members. For example, we could imagine considering the group as being able to distinguish two points if one (or more) of its members can distinguish them. The set of points indistinguishable by $G$ from the current one is then the intersection of the sets of points indistinguishable by the individual members of the group. We can therefore define when a group $G$ has distributed knowledge of a fact $\varphi$ as follows:

(g) $(\mathcal{I}, r, t) \models D_{G} \psi$ iff $\left(\mathcal{I}, r^{\prime}, t^{\prime}\right) \models \psi$ for all $\left(r^{\prime}, t^{\prime}\right)$ in $R$ satisfying $v\left(p_{i}, r, t\right)=v\left(p_{i}, r^{\prime}, t^{\prime}\right)$ for all $p_{i} \in G$.

Notice that indeed under this definition, if one member of $G$ knows $\varphi$ while another member knows that $\varphi \supset \psi$, then the members of $G$ have distributed knowledge of $\psi$. The definition of distributed knowledge given above is in a precise sense a direct 
generalization of the definition of individual processors' knowledge in clause (d) above. We can define the joint view assigned by $v$ to $G$ to be

$$
v(G, r, t) \stackrel{\text { def }}{=}\left\{\left\langle p_{i}, v\left(p_{i}, r, t\right)\right\rangle: p_{i} \in G\right\} .
$$

It is easy to check that $(\mathcal{I}, r, t) \models D_{G} \psi$ iff $\left(\mathcal{I}, r^{\prime}, t^{\prime}\right) \models \psi$ for all $\left(r^{\prime}, t^{\prime}\right)$ in $R$ satisfying $v(G, r, t)=v\left(G, r^{\prime}, t^{\prime}\right)$. Thus, we can identify the distributed knowledge of a group $G$ with the knowledge of an agent whose view is the group's joint view. Note that the knowledge distributed in a group of size one coincides with its unique member's knowledge.

View-based interpretations will prove to be a useful way of ascribing knowledge to processors for the purpose of the design and analysis of distributed protocols. We now discuss some of the basic properties of knowledge in view-based interpretations. Fix a system $R$ and a view function $v$. We can construct a graph corresponding to $R$ and $v$ by taking the nodes of the graph to be all the points of $R$, and joining two nodes $(r, t)$ and $\left(r^{\prime}, t^{\prime}\right)$ by an edge labelled $p_{i}$ if $v\left(p_{i}, r, t\right)=v\left(p_{i}, r^{\prime}, t^{\prime}\right)$; i.e., if $p_{i}$ has the same view at both points. Our definition of knowledge under a view-based interpretation immediately implies that $K_{i} \varphi$ holds at a given point $(r, t)$ if and only if $\varphi$ holds at all points $\left(r^{\prime}, t^{\prime}\right)$ that share an edge labeled $p_{i}$ with $(r, t)$. Define a point $\left(r^{\prime}, t^{\prime}\right)$ in this graph to be $G$-reachable from $(r, t)$ in $k$ steps (with respect to the view function $v$ ) if there exist points $\left(r_{0}, t_{0}\right)$, $\left(r_{1}, t_{1}\right), \ldots,\left(r_{k}, t_{k}\right)$ such that $(r, t)=\left(r_{0}, t_{0}\right),\left(r^{\prime}, t^{\prime}\right)=\left(r_{k}, t_{k}\right)$, and for every $i<k$ there is a processor $p_{j_{i}} \in G$ such that $\left(r_{i}, t_{i}\right)$ and $\left(r_{i+1}, t_{i+1}\right)$ are joined by an edge labeled $p_{j_{i}}$. It follows that $E_{G} \varphi$ holds at $(r, t)$ under this view-based interpretation exactly if $\varphi$ holds at all points $G$-reachable from $(r, t)$ in 1 step. An easy induction on $k$ shows that $E_{G}^{k} \varphi$ holds exactly if $\varphi$ holds at all points $G$-reachable in $k$ steps. Consequently, it is easy to see that $C_{G} \varphi$ holds at a point $(r, t)$ if and only if $\varphi$ holds at all points that are $G$-reachable from $(r, t)$ in a finite number of steps. In the particular case that $G$ is the set of all processors, then $C_{G} \varphi$ holds at $(r, t)$ exactly if $\varphi$ holds at all points in the same connected component of the graph as $(r, t)$.

The way distributed knowledge is represented in this graph is also instructive: $D_{G} \varphi$ holds at a given point $(r, t)$ iff $\varphi$ holds at all points $\left(r^{\prime}, t^{\prime}\right)$ such that for each $p_{i} \in G$, there is an edge between $(r, t)$ and $\left(r^{\prime}, t^{\prime}\right)$ labelled by $p_{i}$. Thus, for distributed knowledge the set of points we need to consider is the intersection of the sets of points we consider when determining what facts each individual processor knows.

By describing the various notions of knowledge in the view-based case via this graph, it becomes easier to investigate their properties. In fact, this graph is very closely related to Kripke structures, a well known standard way of modeling modal logics. In fact, drawing on the theory of modal logics, we can immediately see that the definition of knowledge in view-based interpretations agrees with the well-known modal logic S5 (cf. [HM92]). A modal operator $M$ is said to have the properties of $S 5$ if it satisfies the following axioms and rule of inference:

\footnotetext{
${ }^{3}$ The knowledge ascribed to a set of processes by Chandy and Misra in CM86 essentially corresponds to the distributed knowledge of that set, as defined here. See also PR85, RK86.
} 
A1. The knowledge axiom: $M \varphi \supset \varphi$,

A2. The consequence closure axiom: $M \varphi \wedge M(\varphi \supset \psi) \supset M \psi$,

A3. The positive introspection axiom: $M \varphi \supset M M \varphi$,

A4. The negative introspection axiom: $\neg M \varphi \supset M \neg M \varphi$, and

R1. The rule of necessitation: From $\varphi$ infer $M \varphi$.

Given a knowledge interpretation $\mathcal{I}$ for a system $R$, a fact $\psi$ is said to be valid in the system if it holds at all knowledge points $(\mathcal{I}, r, t)$ for points $(r, t)$ of $R$. In our context the rule $\mathrm{R} 1$ means that whenever $\varphi$ is valid in the system, so is $M \varphi$.

We can now show:

Proposition 1: Under view-based knowledge interpretations, the operators $K_{i}, D_{G}$, and $C_{G}$ all have the properties of S5.

The proof is a consequence of the fact that the definitions of these notions are based on equivalence relations (over points): The relation of processor $p_{i}$ 's having the same view at two points, the relation of all processors in $G$ having the same joint views at both points, and the relation of being reachable via a path consisting solely of edges labeled by members of $G$ in the graph corresponding to the view, are all equivalence relations. The proof of this proposition can be found in HM92.

In addition to having the properties of S5, common knowledge has two additional useful properties under view-based interpretations:

C1. The fixed point axiom: $C_{G} \varphi \equiv E_{G}\left(\varphi \wedge C_{G} \varphi\right)$, and

C2. The induction rule: From $\varphi \supset E_{G}(\varphi \wedge \psi)$ infer $\varphi \supset C_{G} \psi$.

The fixed point axiom essentially characterizes $C_{G} \varphi$ as the solution of a fixed point equation (in fact, it is the greatest solution; we discuss this in more detail in Section 11 and Appendix A). This property of common knowledge is crucial in many of our proofs.

Intuitively, the induction rule says that if $\varphi$ is "public" and implies $\psi$, so that whenever $\varphi$ holds then everybody knows $\varphi \wedge \psi$, then whenever $\varphi$ holds, $\psi$ is common knowledge. We call it the "induction rule" because it is closely related to the notion of induction in arithmetic: Using the fact that $\varphi \supset E_{G}(\varphi \wedge \psi)$ is valid in the system, we can prove by induction on $k$ that $\varphi \supset E_{G}^{k}(\varphi \wedge \psi)$ is also valid in the system, for all $k>0$. It then follows that $\varphi \supset C_{G} \psi$ is valid in the system. Roughly speaking, this proof traces our line of reasoning when we argued that the children in the muddy children puzzle attain common knowledge of the father's statement. We can get an important special case of the Induction Rule by taking $\psi$ to be $\varphi$. Since $E_{G}(\varphi \wedge \varphi)$ is equivalent to $E_{G} \varphi$, we get that from $\varphi \supset E_{G} \varphi$ we can infer $\varphi \supset C_{G} \varphi$. 
A very important instance of view-based knowledge interpretations, that will be used extensively from Section 11 on, is called the complete-history interpretation. Under this interpretation we have $v\left(p_{i}, r, t\right) \stackrel{\text { def }}{=} h\left(p_{i}, r, t\right)$. That is, the processor's complete history is taken to be the view on which the processor's knowledge is based. (In a previous version of this paper HM90, this was called the total view interpretation.) The completehistory interpretation makes the finest possible distinctions among histories. Thus, in a precise sense, it provides the processors with at least as much knowledge about the ground formulas as any other view-based interpretation. This is one of the reasons why the complete-history interpretation is particularly well suited for proving possibility and impossibility of achieving certain goals in distributed systems, and for the design and analysis of distributed protocols (cf. CM86, DM90, MT88).

Notice that view-based knowledge interpretations ascribe knowledge to a processor without the processor necessarily being "aware" of this knowledge, and without the processor needing to perform any particular computation in order to obtain such knowledge. Interestingly, even if the view function $v$ does not distinguish between possibilities at all, that is, if there is a single view $\Lambda$ such that $v\left(p_{i}, r, t\right)=\Lambda$ for all $p_{i}, r$, and $t$, the processors are still ascribed quite a bit of knowledge: every fact that is true at all points of the system is common knowledge among all the processors under this view-based interpretation (and in fact under all view-based interpretations). Note that the hierarchy of Section 3 collapses under this interpretation, with $D \varphi \equiv E \varphi \equiv C \varphi$. This interpretation makes the coarsest possible distinctions among histories; at the other extreme we have the complete-history interpretation, which makes the finest possible distinctions among histories.

Another reasonable view-based interpretation is one in which $v\left(p_{i}, r, t\right)$ is defined to be $p_{i}$ 's local state at $(r, t)$. (Recall that processors are state machines, and are thus assumed to be in some local state at every point). This is the choice made in [FI86, Ros85, RK86. Under this interpretation, a processor might "forget" facts that it knows. In particular, if a processor can arrive at a given state by two different message histories, then, once in that state, the processor's knowledge cannot distinguish between these two "possible pasts". In the complete-history interpretation, a processor's view encodes all of the processor's previous states, and therefore processors do not forget what they know; if a processor knows $\varphi$ at a knowledge point $(\mathcal{I}, r, t)$, then at all knowledge points $\left(\mathcal{I}, r, t^{\prime}\right)$ with $t^{\prime}>t$ the processor will know that it once knew $\varphi$. Thus, while there may be temporary facts such as "it is 3 on my clock" which a processor will not know at 4 o'clock, it will know at 4 o'clock that it previously knew that it was 3 o'clock.

Other view-based interpretations that may be of interest are ones in which a processor's view is identified with the contents of its memory, or with the position of its program counter (see KT86 for a closer look at some of these view-based interpretations). The precise view-based interpretation we choose will vary from application to application. For proving lower bounds we frequently use the complete-history interpretation since, in general, if processors cannot perform an action with the knowledge they have in the complete-history interpretation, they cannot perform it at all. On the other hand, if 
we can show that very little information is required to perform a given action, this may suggest an efficient protocol for performing it.

Although view-based knowledge interpretations are natural and useful in many applications, they do not cover all reasonable possibilities of ascribing knowledge to processors in a distributed system. For example, as we have commented above, view-based knowledge interpretations ascribe knowledge to processors in a fashion that is independent of the processor's computational power. To the extent that we intend processors' knowledge to closely correspond to the actions they can perform, it often becomes crucial to define knowledge in a way that depends on the processors' computational powers (cf. MT88, Mos88). In most of the paper we deal exclusively with view-based knowledge interpretations. However, in order to be able to prove stronger negative results about the attainability of certain states of knowledge, we now give a general definition of knowledge interpretations, which we believe covers all reasonable cases.

Intuitively, we want to allow any interpretation that satisfies the two properties discussed in Section 3: (1) that a processor's knowledge be a function of its history and (2) that only true things be known (so that the axiom $K_{i} \varphi \supset \varphi$ is valid). We capture the first property through the notion of an epistemic interpretation. An epistemic interpretation $\mathcal{I}$ is a function assigning to every processor $p_{i}$ at any given point $(r, t)$, a set $\mathcal{K}_{i}^{\mathcal{I}}(r, t)$ of facts in the extended language that $p_{i}$ is said to "believe". $\mathcal{K}_{i}^{\mathcal{I}}(r, t)$ is required to be a function of $p_{i}$ 's history at $(r, t)$. Thus, if $h\left(p_{i}, r, t\right)=h\left(p_{i}, r^{\prime}, t^{\prime}\right)$, then $\mathcal{K}_{i}^{\mathcal{I}}(r, t)=\mathcal{K}_{i}^{\mathcal{I}}\left(r^{\prime}, t^{\prime}\right)$.

Given an epistemic interpretation $\mathcal{I}$, we now specify when a formula $\varphi$ of the extended language holds at a point $(r, t)$ (denoted $(\mathcal{I}, r, t) \models \varphi)$. As before, if $\varphi$ is a ground fact, we say that $(\mathcal{I}, r, t) \models \varphi$ iff $\pi(r, t)(\varphi)=$ true, while if $\varphi$ is a conjunction or a negation, then its truth is defined based on the truth of its subformulas in the obvious way. If $\varphi$ is of the form $K_{i} \psi$, then $(\mathcal{I}, r, t) \models K_{i} \psi$ iff $\psi \in \mathcal{K}_{i}^{\mathcal{I}}(r, t)$. In this case we say that $p_{i}$ believes $\psi$. The formula $E_{G} \psi$ is identified with the conjunction $\bigwedge_{p_{i} \in G} K_{i} \psi$, so that $(\mathcal{I}, r, t) \models E_{G} \psi$ iff $(\mathcal{I}, r, t) \models K_{i} \psi$ for all $p_{i} \in G$. If $\varphi$ is of the form $C_{G} \psi$, then $(\mathcal{I}, r, t) \models C_{G} \psi$ iff $(\mathcal{I}, r, t) \models E_{G}\left(\psi \wedge C_{G} \psi\right)$. Thus, common knowledge is defined so that the fixed point axiom holds, rather than as an infinite conjunction. Although this definition seems circular, it is not. In order to determine if $(\mathcal{I}, r, t) \models C_{G} \psi$, we first check if $(\mathcal{I}, r, t) \models K_{i}\left(\psi \wedge C_{G} \psi\right)$ for all $p_{i} \in G$. The latter fact can be determined by considering the sets $\mathcal{K}_{i}^{\mathcal{I}}(r, t)$. Finally, to handle distributed knowledge, we need to add a set $\mathcal{K}_{G}^{\mathcal{I}}(r, t)$ of formulas to every point $(r, t)$ for each set of processors $G$, analogous to the sets $\mathcal{K}_{i}^{\mathcal{I}}(r, t)$ for individual processors. We define $(\mathcal{I}, r, t) \models D_{G} \varphi$ if $\varphi \in \mathcal{K}_{G}^{\mathcal{I}}(r, t)$. The sets $\mathcal{K}_{G}^{\mathcal{I}}(r, t)$ must be a function of $G^{\prime}$ 's joint history at $(r, t)$. We may want to put some restrictions on the sets $\mathcal{K}_{G}^{\mathcal{I}}(r, t)$. For example, we may require that if $i \in G$ and $\varphi \in \mathcal{K}_{i}^{\mathcal{I}}(r, t)$ then $\varphi \in \mathcal{K}_{G}^{\mathcal{I}}(r, t)$ (which implies that $K_{i} \varphi \supset D_{G} \varphi$ is valid). Since we do not consider distributed knowledge in interpretations that are not view based, we do not pursue the matter any further here.

The knowledge axiom $K_{i} \varphi \supset \varphi$ is not necessarily valid in epistemic interpretations. Indeed, that is why we have interpreted $K_{i} \varphi$ as "processor $i$ believes $\varphi$ " in epistemic 
interpretations, since the knowledge axiom is the key property that is taken to distinguish knowledge from belief. A processor's beliefs may be false, although a processor cannot be said to know $\varphi$ if $\varphi$ is in fact false. Given an epistemic interpretation $\mathcal{I}$ and a set of runs $R$, we say that $\mathcal{I}$ is a knowledge interpretation for $R$ if for all processors $p_{i}$, times $t$, runs $r \in R$ and formulas $\varphi$ in the extended language, it is the case that whenever $(\mathcal{I}, r, t) \models K_{i} \varphi$ holds, $(\mathcal{I}, r, t) \models \varphi$ also holds. Thus, an epistemic interpretation for $R$ is a knowledge interpretation for $R$ exactly if it makes the knowledge axiom valid in $R$. Notice that the view-based knowledge interpretations defined above are in particular knowledge interpretations.

A trivial consequence of our definitions above is:

Lemma 2: Let $\mathcal{I}$ be a knowledge interpretation for $R$ and let $(r, t)$ be a point of $R$. The following are equivalent for a nonempty subset $G$ of processors:

1. $(\mathcal{I}, r, t) \models C_{G} \varphi$

2. $(\mathcal{I}, r, t) \models K_{i}\left(\varphi \wedge C_{G} \varphi\right)$ for all processors $p_{i} \in G$

3. $(\mathcal{I}, r, t) \models K_{i}\left(\varphi \wedge C_{G} \varphi\right)$ for some processor $p_{i} \in G$.

This lemma shows that common knowledge requires simultaneity in a very strong sense: When a new fact becomes common knowledge in a group $G$, the local histories of all of the members of $G$ must change simultaneously to reflect the event of the fact's becoming common knowledge. This point is perhaps best understood if we think of time as ranging over the natural numbers. Given a knowledge interpretation $\mathcal{I}$, suppose that common knowledge does not hold at the point $(r, t)$ but does hold at the point $(r, t+1)$, so that $(\mathcal{I}, r, t) \models \neg C_{G} \varphi$ and $(\mathcal{I}, r, t+1) \models C_{G} \varphi$. Then it must be the case that the local histories of all processors in $G$ changed between times $t$ and $t+1$. To see this, note that by Lemma 2 we have $(\mathcal{I}, r, t+1) \models K_{i}\left(\varphi \wedge C_{G} \varphi\right)$ for all $p_{i} \in G$. Suppose $p_{i} \in G$ has the same local history in $(r, t)$ and $(r, t+1)$. Then by our assumption that a processor's knowledge depends only on its local history, we have that $(\mathcal{I}, r, t) \models K_{i}\left(\varphi \wedge C_{G} \varphi\right)$. Now by Lemma 2 again, we have $(\mathcal{I}, r, t) \models C_{G} \varphi$, contradicting our original assumption.

We close this section with another trivial observation that follows easily from Lemma 2 .

Lemma 3: Let $\mathcal{I}$ be a knowledge interpretation for $R$, let $r$ and $r^{\prime}$ be runs in $R$, and let $p_{i}$ be a processor in $G$. If $h\left(p_{i}, r, t\right)=h\left(p_{i}, r^{\prime}, t^{\prime}\right)$ then $(\mathcal{I}, r, t) \models C_{G} \varphi$ iff $\left(\mathcal{I}, r^{\prime}, t^{\prime}\right) \models C_{G} \varphi$.

Proof: Given that $p_{i} \in G$, we have by Lemma 2 that $(\mathcal{I}, r, t) \models C_{G} \varphi$ iff $(\mathcal{I}, r, t) \models$ $K_{i}\left(\varphi \wedge C_{G} \varphi\right)$. Since $h\left(p_{i}, r, t\right)=h\left(p_{i}, r^{\prime}, t^{\prime}\right)$, this holds iff $\left(\mathcal{I}, r^{\prime}, t^{\prime}\right) \models K_{i}\left(\varphi \wedge C_{G} \varphi\right)$. Again by Lemma 2 this is true iff $\left(\mathcal{I}, r^{\prime}, t^{\prime}\right) \models C_{G} \varphi$, and we are done. 


\section{Coordinated attack revisited}

Now that we have the basic terminology with which to define distributed systems and knowledge in distributed systems, we can relate the ability to perform a coordinated attack to the attainment of common knowledge of particular facts. This in turn will motivate an investigation of the attainability of common knowledge in systems of various types.

We formalize the coordinated attack problem as follows: We consider the generals as processors and their messengers as communication links between them. The generals are assumed to each behave according to some predetermined deterministic protocol; i.e., a general's actions (what messages it sends and whether it attacks) at a given point are a deterministic function of his history and the time on his clock. In particular, we assume that the generals are following a joint protocol $\left(P_{A}, P_{B}\right)$, where $A$ follows $P_{A}$ and $B$ follows $P_{B}$. We can thus identify the generals with a distributed system $R$, consisting of all possible runs of $\left(P_{A}, P_{B}\right)$. According to the description of the coordinated attack problem in Section 4, the divisions do not initially have plans to attack. Formally, this means that the joint protocol the generals are following has the property that in the absence of any successful communication neither general will attack. Thus, in any run of $R$ where no messages are delivered, the generals do not attack.

We can now show that attacking requires attaining common knowledge of the attack:

Proposition 4: Any correct protocol for the coordinated attack problem has the property that whenever the generals attack, it is common knowledge that they are attacking.

Proof: Let $\left(P_{A}, P_{B}\right)$ be a correct (joint) protocol for the coordinated attack problem, with $R$ being the corresponding system. Consider a ground language consisting of a single fact $\psi \stackrel{\text { def }}{=}$ "both generals are attacking", let $\pi(r, t)$ assign a truth value to this formula in the obvious way at each point $(r, t)$, and let $\mathcal{I}$ be the corresponding completehistory interpretation. Assume that the generals attack at the point $(\hat{r}, \hat{t})$ of $R$. We show that $(\mathcal{I}, \hat{r}, \hat{t}) \models C \psi$. Our first step is to show that $\psi \supset E \psi$ is valid in the system $R$. Assume that $(r, t)$ is an arbitrary point of $R$. If $(\mathcal{I}, r, t) \models \neg \psi$, then we trivially have $(\mathcal{I}, r, t) \models \psi \supset E \psi$. If $(\mathcal{I}, r, t) \models \psi$, then both generals attack at $(r, t)$. Suppose that $\left(r^{\prime}, t^{\prime}\right)$ is a point of $R$ in which $A$ has the same local history as in $(r, t)$. Since $A$ is executing a deterministic protocol and $A$ attacks in $(r, t), A$ must also attack in $\left(r^{\prime}, t^{\prime}\right)$. Furthermore, given that the protocol is a correct protocol for coordinated attack, if $A$ attacks in $\left(r^{\prime}, t^{\prime}\right)$, then so does $B$, and hence $\left(\mathcal{I}, r^{\prime}, t^{\prime}\right) \models \psi$. It follows that $(\mathcal{I}, r, t) \models K_{A} \psi$; similarly we obtain $(\mathcal{I}, r, t) \models K_{B} \psi$. Thus $(\mathcal{I}, r, t) \models E \psi$, and again we have $(\mathcal{I}, r, t) \models \psi \supset E \psi$. We have now shown that $\psi \supset E \psi$ is valid in $R$. By the induction rule it follows that $\psi \supset C \psi$ is also valid in $R$. Since $(\mathcal{I}, \hat{r}, \hat{t}) \models \psi$, we have that $(\mathcal{I}, \hat{r}, \hat{t}) \models C \psi$ and we are done.

Proposition 4 shows that common knowledge is a prerequisite for coordinated attack. Unfortunately, common knowledge is not always attainable, as we show in the next section. Indeed, it is the unattainability of common knowledge that is the fundamental reason why the generals cannot coordinate an attack. 


\section{Attaining common knowledge}

Following the coordinated attack example, we first consider systems in which communication is not guaranteed. Intuitively, communication is not guaranteed in a system if messages might fail to be delivered in an arbitrary fashion, independent of any other event in the system. Completely formalizing this intuition seems to be rather cumbersome (cf. [HF85]), and we do not attempt to do so here. For our purposes, a weak condition, which must be satisfied by any reasonable definition of the notion of communication not being guaranteed, will suffice. Roughly speaking, we take communication not being guaranteed to correspond to two conditions. The first says that it is always possible that from some point on no messages will be received. The second says that if processor $p_{i}$ does not get any information to the contrary (by receiving some message), then $p_{i}$ considers it possible that none of its messages were received.

Formally, given a system $R$, we say that communication in $R$ is not guaranteed if the following two conditions hold:

NG1. For all runs $r$ and times $t$, there exists a run $r^{\prime}$ extending $(r, t)$ such that $r$ and $r^{\prime}$ have the same initial configuration and the same clock readings, and no messages are received in $r^{\prime}$ at or after time $t$.

NG2. If in run $r$ processor $p_{i}$ does not receive any messages in the interval $\left(t^{\prime}, t\right)$, then there is a run $r^{\prime}$ extending $\left(r, t^{\prime}\right)$ such that $r$ and $r^{\prime}$ have the same initial configuration and the same clock readings, $h\left(p_{i}, r, t^{\prime \prime}\right)=h\left(p_{i}, r^{\prime}, t^{\prime \prime}\right)$ for all $t^{\prime \prime} \leq t$, and no processor $p_{j} \neq p_{i}$ receives a message in $r^{\prime}$ in the interval $\left[t^{\prime}, t\right)$.

Note that the requirement that $r$ and $r^{\prime}$ have the same initial configuration already follows from the fact that $r^{\prime}$ extends $(r, t)$ if all the processors have woken up by time $t$ in run $r$. In particular, if we restricted attention to systems where all processors were up at time 0 , we would not require this condition.

We can now show that in a system in which communication is not guaranteed, common knowledge is not attainable.

Theorem 5: Let $R$ be a system in which communication is not guaranteed, let $\mathcal{I}$ be a knowledge interpretation for $R$, and let $|G| \geq 2$. Let $r$ be a run of $R$, and let $r^{-}$be a run of $R$ with the same initial configuration and the same clock readings as $r$, such that no messages are received in $r^{-}$up to time $t$. Then for all formulas $\varphi$ it is the case that $(\mathcal{I}, r, t) \models C_{G} \varphi$ iff $\left(\mathcal{I}, r^{-}, t\right) \models C_{G} \varphi$.

Proof: $\quad$ Fix $\varphi$. Without loss of generality, we can assume $p_{1}, p_{2} \in G$. Let $d(r)$ be the number of messages received in $r$ up to (but not including) time $t$. We show by induction on $k$ that if $d(r)=k$, then $(\mathcal{I}, r, t) \models C_{G} \varphi$ iff $\left(\mathcal{I}, r^{-}, t\right) \models C_{G} \varphi$. We assume that all the runs mentioned in the remainder of the proof have the same initial configuration and the same clock readings as $r$. First assume that $d(r)=0$. Thus no messages are 
received in $r$ up to time $t$. Since $r$ and $r^{-}$have the same initial configuration and clock readings, it follows that $h\left(p_{1}, r, t\right)=h\left(p_{1}, r^{-}, t\right)$. By Lemma 3 we have $\left(\mathcal{I}, r^{-}, t\right) \models C_{G} \varphi$ iff $(\mathcal{I}, r, t) \models C_{G} \varphi$, as desired.

Assume inductively that the claim holds for all runs $r^{\prime} \in R$ with $d\left(r^{\prime}\right)=k$, and assume that $d(r)=k+1$. Let $t^{\prime}<t$ be the latest time at which a message is received in $r$ before time $t$. Let $p_{j}$ be a processor that receives a message at time $t^{\prime}$ in $r$. Let $p_{i}$ be a processor in $G$ such that $p_{i} \neq p_{j}$ (such a $p_{i}$ exists since $|G| \geq 2$ ). From property NG2 in the definition of communication not being guaranteed, it follows that there is a run $r^{\prime} \in R$ extending $\left(r, t^{\prime}\right)$ such that $h\left(p_{i}, r, t^{\prime \prime}\right)=h\left(p_{i}, r^{\prime}, t^{\prime \prime}\right)$ for all $t^{\prime \prime} \leq t$ and all processors $p_{k} \neq p_{i}$ receive no messages in $r^{\prime}$ in the interval $\left[t^{\prime}, t\right)$. By construction, $d\left(r^{\prime}\right) \leq k$, so by the inductive hypothesis we have that $\left(\mathcal{I}, r^{-}, t\right) \models C_{G} \varphi$ iff $\left(\mathcal{I}, r^{\prime}, t\right) \models C_{G} \varphi$. Since $h\left(p_{i}, r, t\right)=h\left(p_{i}, r^{\prime}, t\right)$, by Lemma 3 we have that $\left(\mathcal{I}, r^{\prime}, t\right) \models C_{G} \varphi$ iff $(\mathcal{I}, r, t) \models C_{G} \varphi$. Thus $\left(\mathcal{I}, r^{-}, t\right) \models C_{G} \varphi$ iff $(\mathcal{I}, r, t) \models C_{G} \varphi$. This completes the proof of the inductive step.

Note that Theorem 5 does not say that no fact can become common knowledge in a system where communication is not guaranteed. In a system where communication is not guaranteed but there is a global clock to which all processors have access, then at 5 o'clock it becomes common knowledge that it is 5 o'clock. f However, the theorem does say that nothing can become common knowledge unless it is also common knowledge in the absence of communication. This is a basic property of systems with unreliable communication, and it allows us to prove the impossibility of coordinated attack.

Corollary 6: Any correct protocol for the coordinated attack problem guarantees that neither party ever attacks (!).

Proof: Recall that communication between the generals is not guaranteed (i.e., it satisfies conditions NG1 and NG2 above), and we assume that in the absence of any successful communication neither general will attack. Thus, if we take $\psi$ to be "both generals are attacking", then $C \psi$ does not hold at any point in a run in which no messages are received (since $\psi$ does not hold at any point of that run). Theorem 5 implies that the generals will never attain common knowledge of $\psi$ in any run, and hence by Proposition 4 the generals will never attack.

It is often suggested that for any action for which $C \varphi$ suffices, there is a $k$ such that $E^{k} \varphi$ suffices, as is the case in the muddy children puzzle. The coordinated attack problem shows that this is false. The generals can attain $E^{k} \varphi$ of many facts $\varphi$ for an arbitrarily large $k$ (for example, if the first $k$ messages are delivered). However, simultaneous coordinated attack requires common knowledge (as is shown in Proposition (1); nothing less will do.

\footnotetext{
${ }^{4}$ We remark that the possible presence of some sort of global clock is essentially all that stops us from saying that no fact can become common knowledge if it was not already common knowledge at the beginning of a run. See Proposition 13 in Appendix B and the discussion before it for conditions under which it is the case that no fact can become common knowledge which was not initially common knowledge.
} 
The requirement of simultaneous attack in the coordinated attack problem is a very strong one. It seems that real life generals do not need a protocol that guarantees such a strong condition, and can probably make do with one that guarantees a non-simultaneous attack. We may want to consider weakening this requirement in order to get something that is achievable. In Section 11 we use a variant of the argument used in Corollary 6 to show that no protocol can even guarantee that if one party attacks then the other will eventually attack! On the other hand, a protocol that guarantees that if one party attacks, then with high probability the other will attack is achievable, under appropriate probabilistic assumptions about message delivery. The details of such a protocol are straightforward and left to the reader.

We can prove a result similar to Theorem 5 even if communication is guaranteed, as long as there is no bound on message delivery times. A system $R$ is said to be a system with unbounded message delivery times if condition NG2 of communication not guaranteed holds, and in addition we have:

NG1'. For all runs $r$ and all times $t, u$, with $t \leq u$, there exists a run $r^{\prime}$ extending $(r, t)$ such that $r^{\prime}$ has the same initial configuration and the same clock readings as $r$, and no messages are received in $r^{\prime}$ in the interval $[t, u]$.

Asynchronous systems are often defined to be systems with unbounded message delivery times (for example, in [FLP85]). Intuitively, condition NG1' says that it is always possible for no messages to be received for arbitrarily long periods of time, whereas condition NG1 says that it is always possible for no messages at all to be received from some time on. In some sense, we can view NG1 as the limit case of NG1'. Notice that both systems where communication is not guaranteed and systems with unbounded message delivery times satisfy condition NG2. The proof of Theorem 5 made use only of NG2, not NG1, so we immediately get

Theorem 7: Let $R$ be a system with unbounded message delivery times, let $\mathcal{I}$ be a knowledge interpretation for $R$, and let $|G| \geq 2$. Let $r$ be a run of $R$, and let $r^{-}$be a run of $R$ with the same initial configuration and the same clock readings as $r$, such that no messages are received in $r^{-}$up to time $t$. Then for all formulas $\varphi$ it is the case that $(\mathcal{I}, r, t) \models C_{G} \varphi$ iff $\left(\mathcal{I}, r^{-}, t\right) \models C_{G} \varphi$.

The previous results show that, in a strong sense, common knowledge is not attainable in a system in which communication is not guaranteed or, for that matter, in a system in which communication is guaranteed, but there is no bound on the message delivery times. However, even when all messages are guaranteed to be delivered within a fixed time bound, common knowledge can be elusive. To see this, consider a system consisting of two processors, R2 and D2, connected by a communication link. Moreover, (it is common knowledge that) communication is guaranteed. But there is some uncertainty in message delivery times. For simplicity, let us assume that any message sent from R2 
to D2 reaches D2 either immediately or after exactly $\epsilon$ seconds; furthermore, assume that this fact is common knowledge. Now suppose that at time $t_{S}, \mathrm{R} 2$ sends D2 a message $m$ that does not contain a timestamp, i.e., does not mention $t_{S}$ in any way. The message $m$ is received by D2 at time $t_{D}$. Let $\operatorname{sent}(m)$ be the fact "the message $m$ has been sent". D2 doesn't know sent $(m)$ initially. How does $\{\mathrm{R} 2, \mathrm{D} 2\}$ 's state of knowledge of sent $(m)$ change with time?

At time $t_{D}$, D2 knows sent $(m)$. Because it might have taken $\epsilon$ time units for $m$ to be delivered, R2 cannot be sure that D2 knows $\operatorname{sent}(m)$ before $t_{S}+\epsilon$. Thus, $K_{R} K_{D} \operatorname{sent}(m)$ holds at time $t_{S}+\epsilon$ and no earlier. D2 knows that R2 will not know that D2 knows $\operatorname{sent}(m)$ before $t_{S}+\epsilon$. Because for all D2 knows $m$ may have been delivered immediately (in which case $t_{S}=t_{D}$ ), D2 does not know that R2 knows that D2 knows $\operatorname{sent}(m)$ before $t_{D}+\epsilon$. Since $t_{D}$ might be equal to $t_{S}+\epsilon$, R2 must wait until $t_{S}+2 \epsilon$ before he knows that $t_{D}+\epsilon$ has passed. Thus, $K_{R} K_{D} K_{R} K_{D} \operatorname{sent}(m)$ holds at time $t_{S}+2 \epsilon$ but no earlier. This line of reasoning can be continued indefinitely, and an easy proof by induction shows that before time $t_{S}+k \epsilon$, the formula $\left(K_{R} K_{D}\right)^{k} \operatorname{sent}(m)$ does not hold, while at $t_{S}+k \epsilon$ it does hold. Thus, it "costs" $\epsilon$ time units to acquire every level of "R2 knows that D2 knows". Recall that $C \operatorname{sent}(m)$ implies $\left(K_{R} K_{D}\right)^{k} \operatorname{sent}(m)$ for every $k$. It follows that $C \operatorname{sent}(m)$ will never be attained!

We can capture this situation using our formal model as follows. Let $M I N=\left\lfloor t_{S} / \epsilon\right\rfloor$, and consider the system with a countable set of runs $\left\{r_{i}, r_{i}^{\prime}: i\right.$ an integer with $\left.i \geq-M I N\right\}$. If $i \geq-M I N$, then in run $r_{i}$, R2 sends the message $m$ at time $t_{S}+i \epsilon$ and D2 receives it at the same time. In run $r_{i}^{\prime}$, R2 again sends the message $m$ at time $t_{S}+i \epsilon$, but D2 receives it at time $t_{S}+(i+1) \epsilon$. (Note our choice of $M I N$ guarantees that all messages are sent at time greater than or equal to 0.) If we assume that in fact the message in the example took $\epsilon$ time to arrive, then the run $r_{0}^{\prime}$ describes the true situation. However, it is easy to see that at all times $t, \mathrm{R} 2$ cannot distinguish runs $r_{i}$ and $r_{i}^{\prime}$ (in that its local state is the same at the corresponding points in the two runs, assuming that only message $m$ is sent), while D2 cannot distinguish $r_{i}$ and $r_{i-1}^{\prime}$ (provided $\left.i-1 \geq-M I N\right)$.

Our discussion of knowledge in a distributed system is motivated by the fact that we can view processors' actions as being based on their knowledge. Consider an eager epistemic interpretation $\mathcal{I}$ under which $\mathrm{R} 2$ believes $C \operatorname{sent}(m)$ as soon as it sends the message $m$, while D2 believes $C \operatorname{sent}(m)$ as soon as it receives $m$. Clearly, $\mathcal{I}$ is not a knowledge interpretation, because it is not knowledge consistent (R2 might believe that D2 knows sent $(m)$, when in fact D2 does not). However, once D2 receives $m$, which happens at most $\epsilon$ time units after R2 starts believing $C \operatorname{sent}(m)$, it is easy to see that $C$ sent $(m)$ does indeed hold! In a sense, Lemma 2 says that attaining common knowledge requires a certain kind of "natural birth"; it is not possible to attain it consistently unless simultaneity is attainable. But if one is willing to give up knowledge consistency (i.e., abandon the $K_{i} \varphi \supset \varphi$ axiom) for short intervals of time, something very similar to common knowledge can be attained.

The period of up to $\epsilon$ time units during which R2 and D2's "knowledge" might be in- 
consistent might have many negative consequences. If the processors need to act based on whether $C \operatorname{sent}(m)$ holds during that interval, they might not act in an appropriately coordinated way. This is a familiar problem in the context of distributed database systems. There, committing a transaction roughly corresponds to entering into an agreement that the transaction has taken place in the database. However, in general, different sites of the database commit transactions at different times (although usually all within a small time interval). When a new transaction is being committed there is a "window of vulnerability" during which different sites might reflect inconsistent histories of the database. However, once all sites commit the transaction, the history of the database that the sites reflect becomes consistent (at least as far as the particular transaction is concerned). In Section 13 we return to the question of when an "almost knowledge consistent" version of common knowledge can be safely used "as if it were" common knowledge.

Returning to the R2-D2 example, note that it is the uncertainty in relative message delivery time that makes it impossible to attain common knowledge, and not the fact that communication is not instantaneous. If it were common knowledge that messages took exactly $\epsilon$ time units to arrive, then $\operatorname{sent}(m)$ would be common knowledge at time $t_{S}+\epsilon$ (and the system would consist only of run $r_{1}$ ).

Another way of removing the uncertainty is by having a common (global) clock in the system. Suppose that there is such a clock. Consider what would happen if R2 sends D2 the following message $m^{\prime}$ :

"This message is being sent at time $t_{S} ; m . "$

Since there is a global clock and it is guaranteed that every message sent by R2 is delivered within $\epsilon$ time units, the fact that R2 sent $m^{\prime}$ to D2 would again become common knowledge at time $t_{S}+\epsilon$ ! In this case, the system would consist of two runs, $r_{0}$ and $r_{1}$. At time $t_{S}+\epsilon$, D2 would know which of the two was actually the case, although R2 would not (although D2 could tell him by sending a message).

It seems that common knowledge is attainable in the latter two cases due to the possibility of simultaneously making the transition from not having common knowledge to having common knowledge (at time $t_{S}+\epsilon$ ). The impossibility of doing so in the first case was the driving force behind the extra cost in time incurred in attaining each additional level of knowledge.

Lemma 1 already implies that when $C \varphi$ first holds all processors must come to believe $C \varphi$ simultaneously. In particular, this means that all of the processors' histories must change simultaneously. However, strictly speaking, practical systems cannot guarantee absolute simultaneity. In particular, we claim that essentially all practical distributed systems have some inherent temporal uncertainty. There is always some uncertainty about the precise instant at which each processor starts functioning, and about exactly how much time each message takes to be delivered. In Appendix B we give a precise formulation of the notion of temporal imprecision, which captures these properties, and use methods derived from [DHS86] and [HMM85] to prove the following result: 
Theorem 8: Let $R$ be a system with temporal imprecision, let $\mathcal{I}$ be a knowledge interpretation for $R$, and let $|G| \geq 2$. Then for all runs $r \in R$, times $t$, and formulas $\varphi$ it is the case that $(\mathcal{I}, r, t) \models C_{G} \varphi$ iff $(\mathcal{I}, r, 0) \models C_{G} \varphi$.

Since practical systems turn out to have temporal imprecision, Theorem 8 implies that, strictly speaking, common knowledge cannot be attained in practical distributed systems! In such systems, we have the following situation: a fact $\varphi$ can be known to a processor without being common knowledge, or it can be common knowledge (in which case that processor also knows $\varphi$ ), but due to (possibly negligible) imperfections in the system's state of synchronization and its communication medium, there is no way of getting from the first situation to the second! Note that if there is a global clock, then there cannot be any temporal imprecision. Thus, it is consistent with Theorem 8 that common knowledge is attainable in a system with a global clock.

Observe that we can now show that, formally speaking, even people cannot attain common knowledge of any new fact! Consider the father publicly announcing $\mathbf{m}$ to the children in the muddy children puzzle. Even if we assume that it is common knowledge that the children all hear whatever the father says and understand it, there remains some uncertainty as to exactly when each child comes to know (or comprehend) the father's statement. Thus, it is easy to see that the children do not immediately have common knowledge of the father's announcement. Furthermore, for similar reasons the father's statement can never become common knowledge.

\section{$9 \quad$ A paradox?}

There is a close correspondence between agreements, coordinated actions, and common knowledge. We have argued that in a precise sense, reaching agreements and coordinating actions in a distributed system requires attaining common knowledge of certain facts. However, in the previous section we showed that common knowledge cannot be attained in practical distributed systems! We are faced with a seemingly paradoxical situation on two accounts. First of all, these results are in contradiction with practical experience, in which operations such as reaching agreement and coordinating actions are routinely performed in many actual distributed systems. It certainly seems as if these actions are performed in such systems without the designers having to worry about common knowledge (and despite the fact that we have proved that common knowledge is unattainable!). Secondly, these results seem to contradict our intuitive feeling that common knowledge is attained in many actual situations; for example, by the children in the muddy children puzzle.

Where is the catch? How can we explain this apparent discrepancy between our formal treatment and practical experience? What is the right way to interpret our negative results from the previous section? Is there indeed a paradox here? Or perhaps we are using a wrong or useless definition of common knowledge?

We believe that we do have a useful and meaningful definition of common knowledge. 
However, a closer inspection of the situation is needed in order to understand the subtle issues involved. First of all, we shall see that only rather strong notions of coordination in a distributed system require common knowledge. Common knowledge corresponds to absolutely simultaneous coordination, which is more than is necessary in many particular applications. For many other types of coordination, weaker states of knowledge suffice. In the coming sections we investigate a variety of weaker states of knowledge that are appropriate for many applications. Furthermore, in many cases practical situations (and practical distributed systems) can be faithfully modeled by a simplified abstract model, in which common knowledge is attainable. In such a case, when facts become common knowledge in the abstract model it may be perfectly safe and reasonable to consider them to be common knowledge when deciding on actions to be performed in the actual system. We discuss this in greater detail in Section 13.

\section{Common knowledge revisited}

In Section 8 we showed that common knowledge is not attainable in practical distributed systems under any reasonable interpretation of knowledge (i.e., in any epistemic interpretation). Our purpose in the coming sections is to investigate what states of knowledge are attainable in such systems. For that purpose, we restrict our attention to view-based interpretations of knowledge, since they seem to be the most appropriate for many applications in distributed systems. Under view-based interpretations, it seems useful to consider an alternative view of common knowledge.

Recall the children's state of knowledge of the fact $\mathbf{m}$ in the muddy children puzzle. If we assume that it is common knowledge that all children comprehend $\mathbf{m}$ simultaneously, then after the father announces $\mathbf{m}$, the children attain $C \mathbf{m}$. However, when they attain $C \mathbf{m}$ it is not the case that the children learn the infinitely many facts of the form $E^{k} \mathbf{m}$ separately. Rather, after the father speaks, the children are in a state of knowledge $S$ characterized by the fact that every child knows both that $\mathbf{m}$ holds and that $S$ holds. Thus, $S$ satisfies the equation

$$
S \equiv E(\mathbf{m} \wedge S)
$$

The fixed point axiom of Section 6 says that under a view-based interpretation, $C_{G} \varphi$ is a solution for $X$ in an analogous fixed point equation, namely

$$
X \equiv E_{G}(\varphi \wedge X)
$$

Now this equation has many solutions, including, for example, both false and $C_{G}(\varphi \wedge$ $\psi$ ), for any formula $\psi \cdot C_{G} \varphi$ can be characterized as being the greatest fixed point of the equation; i.e., a fixed point that is implied by all other solutions. (The least fixed point of this equation is false, since it implies all other solutions.) As our discussion of common knowledge in the case of the muddy children puzzle suggests, expressing common knowledge as a greatest fixed point of such an equation seems to correspond more closely 
to the way it actually arises. We sketch a semantics for a propositional view-based logic of knowledge with fixed points in Appendix A. This alternative point of view, considering common knowledge as the greatest fixed point of such an equation, will turn out to be very useful when we attempt to define related variants of common knowledge.

\section{$11 \epsilon$-common knowledge and $\diamond$-common knowledge}

Since, strictly speaking, common knowledge cannot be attained in practical distributed systems, it is natural to ask what states of knowledge can be obtained by the communication process. In this section we consider what states of knowledge are attained in systems in which communication delivery is guaranteed but message delivery times are uncertain. For ease of exposition, we restrict our attention to view-based interpretations of knowledge here and in the next section.

We begin by considering synchronous broadcast channels of communication; i.e., ones where every message sent is received by all processors, and there are constants $L$ and $\epsilon$ such that all processors receive the message between $L$ and $L+\epsilon$ time units from the time it is sent. We call $\epsilon$ the broadcast spread of such a channel. Recall that the properties of the system hold throughout all of its runs and hence are common knowledge. In particular, the properties of the broadcast channel are common knowledge under any view-based interpretation.

Let us now consider the state of knowledge of the system when a processor $p_{i}$ receives a broadcast message $m$. Clearly $p_{i}$ knows that within an interval of $\epsilon$ time units around the current time everyone (receives $m$ and) knows $\operatorname{sent}(m)$. But $p_{i}$ also knows that any other processor that receives $m$ will know that all processors will receive $m$ within such an $\epsilon$ interval. Let us define within an $\epsilon$ interval, everyone knows $\varphi$, denoted $E^{\epsilon} \varphi$, to hold if there is an interval of $\epsilon$ time units containing the current time such that each processor comes to know $\varphi$ at some point in this interval. Formally, we have: $(\mathcal{I}, r, t) \models E_{G}^{\epsilon} \varphi$ if there exists an interval $I=\left[t^{\prime}, t^{\prime}+\epsilon\right]$ such that $t \in I$ and for all $p_{i} \in G$ there exists $t_{i} \in I$ for which $\left(\mathcal{I}, r, t_{i}\right) \models K_{i} \varphi$. Let $\psi$ be "some processor has received $m$ ". In a synchronous broadcast system as described above, we clearly have that $\psi \supset E^{\epsilon} \psi$ is valid.

We are thus in a state of knowledge that is analogous to common knowledge; here, however, rather than everyone knowing $\varphi$ at the same instant, they all come to know $\varphi$ within an interval of $\epsilon$ time units. We call this the state of group knowledge $\epsilon$-common knowledge, denoted $C^{\epsilon}$. The formal definition of $C_{G}^{\epsilon} \varphi$ is as the greatest fixed point of the equation:

$$
X \equiv E_{G}^{\epsilon}(\varphi \wedge X)
$$

We refer the reader to Appendix A for a rigorous definition. The fact $\psi$ above, stating that some processor received the message $m$, has the property that $\psi \supset C^{\epsilon} \psi$. In addition, as $\psi \supset \operatorname{sent}(m)$ is valid, it is also the case that $\psi \supset C^{\epsilon} \operatorname{sent}(m)$. Thus, when some processor receives $m$ it becomes $\epsilon$-common knowledge that $m$ has been sent. 
As a straightforward consequence of its definition, $C^{\epsilon}$ satisfies the appropriate analogues of the fixed point axiom $\mathrm{C} 1$ and the induction rule $\mathrm{C} 2$ of Section 6 (replacing $E$ by $E^{\epsilon}$ and $C$ by $C^{\epsilon}$ ). Note that we did not define $C_{G}^{\epsilon} \varphi$ as an infinite conjunction of $\left(E_{G}^{\epsilon}\right)^{k} \varphi, k \geq 1$. While it is not hard to show that $C_{G}^{\epsilon} \varphi$ implies this infinite conjunction, it is not equivalent to it; however, giving a detailed counterexample is beyond the scope of this paper. (We give an example of a similar phenomenon below.) The fixed point definition is the one that is appropriate for our applications. Just as common knowledge corresponds to simultaneous actions in a distributed system, $\epsilon$-common knowledge corresponds to actions that are guaranteed to be performed within $\epsilon$ time units of one another. This is what we get from the fixed point axiom $\mathrm{C}$, which does not hold in general for the infinite conjunction. We are often interested in actions that are guaranteed to be performed within a small time window. For example, in an "early stopping" protocol for Byzantine agreement (cf. [DRS90]), all correct processors are guaranteed to decide on a common value within $\epsilon$ time units of each other. It follows that once the first processor decides, the decision value is $\epsilon$-common knowledge.

There is one important special case where it can be shown that the fixed point definition of $C_{G}^{\epsilon} \varphi$ is equivalent to the infinite conjunction. This arises when we restrict attention to complete-history interpretations and stable facts, facts that once true, remain true. Many facts of interest in distributed systems applications, such as " $\varphi$ held at some point in the past", "the initial value of $x$ is 1 ", or " $\varphi$ holds at time $t$ on $p_{i}$ 's clock", are stable. If $\varphi$ is stable, then it is not hard to check that in complete-history interpretations, we have that $E_{G}^{\epsilon} \varphi$ holds iff $E_{G} \varphi$ will hold in $\epsilon$ time units. As a straightforward consequence of this observation, we can show that in complete-history interpretations, for a stable fact $\varphi$ we do have that $C_{G}^{\epsilon} \varphi$ holds iff $\left(E_{G}^{\epsilon}\right)^{k} \varphi$ holds for all $k \geq 1$.

It is not hard to verify that of the properties of S5, $C^{\epsilon}$ satisfies only A3 (positive introspection) and R1 (the rule of necessitation). The failure of $C^{\epsilon}$ to satisfy the knowledge axiom and the consequence closure axiom can be traced to the failure of $E^{\epsilon}$ to satisfy these axioms. The problem is that $E^{\epsilon} \varphi$ only requires that $\varphi$ hold and be known at some (not all!) of the points in the $\epsilon$ interval $I$. Indeed, it is not hard to construct an example in which $E^{\epsilon} \varphi \wedge E^{\epsilon} \neg \varphi$ holds. We remark that if we restrict attention to stable facts and complete-history interpretations, then consequence closure does hold for both $E^{\epsilon}$ and $C^{\epsilon}$

It is interesting to compare $\epsilon$-common knowledge with common knowledge. Clearly, $C \varphi \supset C^{\epsilon} \varphi$ is valid. However, since synchronous broadcast channels are implementable in systems where common knowledge is not attainable, the converse does not hold. Thus, $\epsilon$-common knowledge is strictly weaker than common knowledge. Moreover, note that while $C \varphi$ is a static state of knowledge, which can be true of a point in time irrespective

\footnotetext{
${ }^{5}$ The situation there is in fact slightly more complicated since only the correct processors are required to decide; see MT88 for definitions of knowledge appropriate for such situations.

${ }^{6}$ We remark that in earlier versions of this paper, we restricted attention to complete-history interpretations and stable facts, and defined $E_{G}^{\epsilon} \varphi$ as $\bigcirc^{\epsilon} E_{G} \varphi$, where $\bigcirc^{\epsilon} \psi$ is true at a point $(r, t)$ iff $\psi$ is true $\epsilon$ time units later, at $(r, t+\epsilon)$. By the comments above, our current definition is a generalization of our former definition.
} 
of its past or future, $C^{\epsilon} \varphi$ is a notion that is essentially temporal. Whether or not it holds depends on what processors will know in an $\epsilon$ interval around the current time.

For any message $m$ broadcast on a channel with broadcast spread $\epsilon$, the fact $\operatorname{sent}(m)$ becomes $\epsilon$-common knowledge $L$ time units after $m$ is broadcast (in particular, as soon as it is sent if $L=0$ ). Upon receiving $m$, a processor $p_{i}$ knows that $C^{\epsilon} \operatorname{sent}(m)$ holds, i.e. $K_{i} C^{\epsilon} \operatorname{sent}(m)$ holds. Returning to R2 and D2's communication problem, we can view them as a synchronous broadcast system, and indeed they attain $C^{\epsilon} \operatorname{sent}(m)$ immediately when $\mathrm{R} 2$ sends the message $m$. (Note that $L=0$ in this particular example; the interested reader is invited to check that $\mathrm{R} 2$ and $\mathrm{D} 2$ in fact achieve $\epsilon / 2$-common knowledge of $\operatorname{sent}(m)$ at time $t_{S}+\epsilon / 2$.)

Having discussed states of knowledge in synchronous broadcast channels, we now turn our attention to systems in which communication is asynchronous: no bound on the delivery times of messages in the system exists. Consider the state of knowledge of $\operatorname{sent}(m)$ in a system in which $m$ is broadcast over an asynchronous channel: a channel that guarantees that every message broadcast will eventually reach every processor. Upon receiving $m$, a processor knows sent $(m)$, and knows that every other processor either has already received $m$ or will eventually receive $m$. This situation, where it is common knowledge that if $m$ is sent then everyone will eventually know that $m$ has been sent, gives rise to a weak state of group knowledge which we call eventual common knowledge.

We define everyone in $G$ will eventually have known $\varphi$, denoted $E_{G}^{\diamond} \varphi$, to hold if for every processor in $G$ there is some time during the run at which it knows $\varphi$. Formally, $(\mathcal{I}, r, t) \models E_{G}^{\diamond} \varphi$ if for all $p_{i} \in G$ there exists $t_{i} \geq 0$ such that $\left(\mathcal{I}, r, t_{i}\right) \models K_{i} \varphi$. We remark that if we restrict attention to stable facts $\varphi$ and complete-history interpretations, then $E_{G}^{\diamond} \varphi$ is equivalent to $\diamond E_{G} \varphi$, that is, eventually everyone in $G$ knows $\varphi$. We define the state of $\diamond$-common knowledge (read eventual common knowledge), denoted by $C^{\diamond}$, by taking $C_{G}^{\diamond} \varphi$ to be the greatest fixed point of the equation:

$$
X \equiv E_{G}^{\diamond}(\varphi \wedge X)
$$

Notice that we again used the fixed point definition rather than one in terms of infinite conjunction of $\left(E_{G}^{\diamond}\right)^{k} \varphi, k \geq 1$. Our definition implies the infinite conjunction but, as we show by example below, it is not equivalent to the infinite conjunction, even if we restrict to stable facts and complete-history interpretations.

Our motivation for considering the fixed point definition is the same as it was in the case of $\epsilon$-common knowledge. The fixed point definition gives us analogues to $\mathrm{C} 1$ and $\mathrm{C} 2$; as a consequence, $\diamond$-common knowledge corresponds to events that are guaranteed to take place at all sites eventually. For example, in some of the work on variants of the Byzantine Agreement problem discussed in the literature (cf. [DRS90), the kind of agreement sought is one in which whenever a correct processor decides on a given value,

\footnotetext{
${ }^{7}$ Formally, we take $\diamond \psi$ to be true at a point $(r, t)$ if $\psi$ is true at some point $\left(r, t^{\prime}\right)$ with $t^{\prime} \geq t$. In an earlier version of this paper, we defined $E_{G}^{\diamond} \varphi$ as $\diamond E_{G} \varphi$. Again, by the comments above, our current definition is a generalization of our former one.
} 
each other correct processor is guaranteed to eventually decide on the same value. The state of knowledge of the decision value that the processors attain in such circumstances is $\diamond$-common knowledge. Also, in asynchronous error-free broadcast channels, a processor knows that $\operatorname{sent}(m)$ is $\diamond$-common knowledge when it receives the message $m$.

$C_{G}^{\diamond}$ is the weakest temporal notion of common knowledge that we have introduced. In fact, we now have a hierarchy of the temporal notions of common knowledge. For any fact $\varphi$ and $\epsilon_{1} \leq \cdots \leq \epsilon_{k} \leq \epsilon_{k+1} \leq \cdots$, we have:

$$
C_{G} \varphi \supset C_{G}^{\epsilon_{1}} \varphi \supset \cdots \supset C_{G}^{\epsilon_{k}} \varphi \supset C_{G}^{\epsilon_{k+1}} \varphi \supset \cdots \supset C_{G}^{\diamond} \varphi
$$

We next consider how $C^{\epsilon}$ and $C^{\diamond}$ are affected by communication not being guaranteed. Recall that Theorem 5 implies that if communication is not guaranteed, then common knowledge is independent of the communication process. A fact only becomes common knowledge if it becomes common knowledge in the absence of messages. Interestingly, the obvious analogue of Theorem 5 does not hold for $C^{\epsilon}$ and $C^{\diamond}$. Indeed, it is possible to construct a situation in which $C^{\epsilon} \varphi$ is attained only if communication is not sufficiently successful. For example, consider a system consisting of R2 and D2 connected by a two-way link. Communication along the link is not guaranteed, R2 and D2's clocks are perfectly synchronized, and both of them run the following protocol: At time 0, send the message "OK". For all natural numbers $k>0$, if you have received $k$ "OK" messages by time $k$ on your clock, send an "OK" message at time $k$; otherwise, send nothing. Let $\psi=$ "it is time $k$ where $k \geq 1$ and some message sent at or before time $k-1$ was not delivered within one time unit." Assume a complete-history interpretation for this system and fix $\epsilon=1$. It is easy to see that $\psi \supset E^{\epsilon} \psi$ is valid in this system. For suppose that at time $k$ the fact $\psi$ holds because one of R2's messages was not delivered to D2. D2 knows $\psi$ at time $k$ and, according to the protocol, will not send a message to $\mathrm{R} 2$ at time $k$. Thus, by time $k+1, \mathrm{R} 2$ will also know $\psi$ (if it didn't know it earlier). The induction rule implies that $\psi \supset C^{\epsilon} \psi$ is also valid in the system. If $r$ is a run of the system where no messages are received, then it is easy to see that $\psi$ holds at $(r, 1)$, and hence so does $C^{\epsilon} \psi$. However, $C^{\epsilon} \psi$ does not hold at $\left(r^{\prime}, 1\right)$ if $r^{\prime}$ is a run where all messages are delivered within one time unit. (The same example works for $C^{\diamond} \psi$.)

In the example above, successful communication in a system where communication is not guaranteed can prevent $C_{G}^{\epsilon} \psi$ (resp. $C_{G}^{\diamond} \psi$ ) from holding. However, the following theorem shows that we can get a partial analogue to Theorem 5 for $C^{\epsilon}$ and $C^{\diamond}$. Intuitively, it states that if $C_{G}^{\epsilon} \psi$ (resp. $C_{G}^{\diamond} \psi$ ) does not hold in the absence of successful communication, then $C_{G}^{\epsilon} \psi$ (resp. $C_{G}^{\diamond} \psi$ ) does not hold regardless of how successful communication may turn out to be. More formally,

Theorem 9: Let $R$ and $G$ be as in Theorem 5, and let $\mathcal{I}$ be a view-based interpretation. Let $r^{-}$be a run of $R$ where no messages are received. If $\left(\mathcal{I}, r^{-}, t\right) \not \forall C_{G}^{\epsilon} \varphi\left(\operatorname{resp} .\left(\mathcal{I}, r^{-}, t\right) \not \models\right.$ $\left.C_{G}^{\diamond} \varphi\right)$ for all times $t$, then $(\mathcal{I}, r, t) \not \forall C_{G}^{\epsilon} \varphi\left(\operatorname{resp} .(\mathcal{I}, r, t) \not C_{G}^{\diamond} \varphi\right)$ for all runs $r$ with the same initial configuration and the same clock readings as $r^{-}$and all times $t$. 
Proof: We sketch the proof for $C_{G}^{\epsilon} \varphi$; the proof for $C_{G}^{\diamond} \varphi$ is analogous. We assume that all runs mentioned in this proof have the same initial configuration and the same clock readings as $r^{-}$. If $r$ is a run such that $C_{G}^{\epsilon} \varphi$ holds at some point in $r$, let $t_{j}(r)$ be the first time in $r$ that processor $p_{j} \in G$ knows $C_{G}^{\epsilon} \varphi$. Let $\hat{t}(r)=\max \left\{t_{j}(r): p_{j} \in G\right\}$, and let $d(r)$ be the number of messages that are received in $r$ up to (but not including) $\hat{t}(r)$. We show by induction on $k$ that if $r$ is a run such that $C_{G}^{\epsilon} \varphi$ holds at some point in $r$, then $d(r) \neq k$. This will show that in fact $C_{G}^{\epsilon} \varphi$ can never hold.

If $d(r)=0$ and $C_{G}^{\epsilon} \varphi$ holds at some point in $r$, choose some $p_{i} \in G$ and let $t_{i}=t_{i}(r)$. Then we have that $\left(\mathcal{I}, r, t_{i}\right) \models K_{i} C_{G}^{\epsilon} \varphi$. Clearly $h\left(p_{i}, r, t_{i}\right)=h\left(p_{i}, r^{-}, t_{i}\right)$, so $\left(\mathcal{I}, r^{-}, t_{i}\right) \models$ $K_{i} C_{G}^{\epsilon} \varphi$. By the knowledge axiom, we have that $\left(\mathcal{I}, r^{-}, t_{i}\right) \models C_{G}^{\epsilon} \varphi$, contradicting the hypothesis of the theorem.

For the inductive step, assume that $d(r)=k+1$ and let $\hat{t}=\hat{t}(r)$. We now proceed as in the proof of Theorem 5 . Let $p_{j}$ be a processor receiving the last message received in $r$ before time $\hat{t}$. Let $t^{\prime}$ be the time at which $p_{j}$ receives this message. Let $p_{i}$ be a processor in $G$ such that $p_{i} \neq p_{j}$ and let $t_{i}=t_{i}(r)$. Since communication is not guaranteed, there exists a run $r^{\prime}$ extending $\left(r, t^{\prime}\right)$ such that (1) no messages are received in $r^{\prime}$ at or after time $\hat{t},(2) h\left(p_{i}, r, t^{\prime \prime}\right)=h\left(p_{i}, r^{\prime}, t^{\prime \prime}\right)$ for all $t^{\prime \prime} \leq \hat{t}$, and (3) all processors $p_{k} \neq p_{i}$ receive no messages in the interval $\left[t^{\prime}, \hat{t}\right)$. By construction, at most $k$ messages are received altogether in $r^{\prime}$, so $d\left(r^{\prime}\right) \leq k$. By the induction hypothesis we have that $\left(\mathcal{I}, r^{\prime}, t^{\prime \prime}\right) \models \neg C_{G}^{\epsilon} \varphi$ for all $t^{\prime \prime}$. It follows that $\left(\mathcal{I}, r^{\prime}, t_{i}\right) \models \neg K_{i} C_{G}^{\epsilon} \varphi$. But since we assumed $\left(\mathcal{I}, r, t_{i}\right) \models K_{i} C_{G}^{\epsilon} \varphi$ and $h\left(p_{i}, r, t_{i}\right)=h\left(p_{i}, r^{\prime}, t_{i}\right)$, this gives us a contradiction.

We can now use Theorem 9 to prove an analogue to Corollary 6 , which shows that if communication is not guaranteed, then there is no protocol for eventually coordinated attack.

Proposition 10: In the coordinated attack problem, any protocol that guarantees that whenever either party attacks the other party will eventually attack, is a protocol in which necessarily neither party attacks.

Proof: The proof is analogous to that of Corollary 6. Assume that $\left(P_{A}, P_{B}\right)$ is a joint protocol that guarantees that if either party attacks then they both eventually attack, and let $R$ be the corresponding system. Let $\psi=$ "At least one of the generals has started attacking". We first show that when either general attacks, then eventual common knowledge of $\psi$ must hold. Since the protocol guarantees that whenever one general attacks the other one eventually attacks, it is easy to see that a general that has decided to attack knows $\psi$ and knows that eventually both generals will know $\psi$. Thus, by the induction rule for $C^{\diamond}$, when a general attacks $C^{\diamond} \psi$ holds. Since in every run of the protocol in which no messages are received no party attacks (and hence neither $\psi$ nor $C^{\diamond} \psi$ hold in such runs), by Theorem 9 , the protocol $\left(P_{A}, P_{B}\right)$ guarantees that neither general will ever attack.

Theorem 9 allows us to construct an example in which the infinite conjunction of $\left(E^{\diamond}\right)^{k} \varphi$ holds, but $C^{\diamond} \varphi$ does not. In the setting of the coordinated attack problem, 
Let $\varphi$ be "General $A$ is in favor of attacking". Consider a run in which all messengers arrive safely, and messages are acknowledged ad infinitum. Clearly, assuming a completehistory interpretation, for all $k$ it is the case that $E^{k} \varphi$ holds after the $k$ th message is delivered. It follows that $\left(E^{\diamond}\right)^{k} \varphi$ holds at time 0 . However, Theorem 9 implies that $C^{\diamond} \varphi$ never holds in this run. It follows that $C^{\diamond} \varphi$ is not equivalent to the infinite conjunction of $\left(E^{\diamond}\right)^{k} \varphi$ even in the case of stable facts $\varphi$ and complete-history interpretations.

Recall that the proof that unreliable communication cannot affect what facts are common knowledge carried over to (reliable) asynchronous communication. Our proof in Theorem 9 clearly does not carry over. In fact, a message broadcast over a reliable asynchronous channel does become eventual common knowledge. However, it is possible to show that asynchronous channels cannot be used in order to attain $\epsilon$-common knowledge:

Theorem 11: Let $R$ be a system with unbounded delivery times and let $|G| \geq 2$. Suppose there is some run $r^{-}$in $R$ in which no message are delivered in the interval $[0, t+\epsilon)$ such that $\left(\mathcal{I}, r^{-}, t\right) \not C_{G}^{\epsilon} \psi$. Then for all runs $r$ in $R$ with the same initial configuration and the same clock readings as $r^{-}$, we have $(\mathcal{I}, r, t) \not \models C_{G}^{\epsilon} \psi$.

Sketch of Proof: The proof essentially follows the proof of Theorems 1 and 9 . We proceed by induction on $d(r)$, the number of messages received in $r$ up to time $t$. Details are left to the reader.

Thus, asynchronous communication channels are of no use for coordinating actions that are guaranteed to be performed at all sites within a predetermined fixed time bound.

\section{Timestamping: using relativistic time}

Real time is not always the appropriate notion of time to consider in a distributed system. Processors in a distributed system often do not have access to a common source of real time, and their clocks do not show identical readings at any given real time. Furthermore, the actions taken by the processors rarely actually depend on real time. Rather, time is often used mainly for correctly sequencing events at the different sites and for maintaining "consistent" views of the state of the system. In this section we consider states of knowledge relative to relativistic notions of time.

Consider the following scenario: R2 knows that R2 and D2's clock differ by at most $\delta$, and that any message R2 sends D2 will arrive within $\epsilon$ time units. R2 sends D2 the following message $m^{\prime}$ :

"This message is being sent at $t_{S}$ on R2's clock, and will reach D2 by $t_{S}+\epsilon+\delta$ on both clocks; $m . "$

Let us denote $t_{S}+\epsilon+\delta$ by $T_{0}$. Now, at time $T_{0}$ on his clock, R2 would like to claim that $\operatorname{sent}\left(m^{\prime}\right)$ is common knowledge. Is it? Well, we know by now that it is not, but it 
is interesting to analyze this situation. Before we do so, let us introduce a relativistic formalism for knowledge, which we call timestamped knowledge: We denote "at time $T$ on his clock, $p_{i}$ knows $\varphi^{\prime \prime}$ by $K_{i}^{T} \varphi$. $T$ is said to be the timestamp associated with this knowledge. We then define

$$
E_{G}^{T} \varphi \equiv \bigwedge_{p_{i} \in G} K_{i}^{T} \varphi
$$

$E^{T} \varphi$ corresponds to everyone knowing $\varphi$ individually at time $T$ on their own clocks. Notice that for $T_{0}$ as above, $\operatorname{sent}\left(m^{\prime}\right) \supset E^{T 0} \operatorname{sent}\left(m^{\prime}\right)$. It is natural to define the corresponding relativistic variant of common knowledge, $C^{T}$, which we call timestamped common knowledge, so that $C_{G}^{T} \varphi$ is the greatest fixed point of the equation

$$
X \equiv E_{G}^{T}(\varphi \wedge X)
$$

So, in any run where the message $m^{\prime}$ is sent, R2 and D2 have timestamped common knowledge of $\operatorname{sent}\left(m^{\prime}\right)$ with timestamp $T_{0}$. It is easy to check that $C^{T}$ satisfies the fixed point axiom and the induction rule, as well as all of the axioms of S5 except for the knowledge axiom. In this respect, $C^{T}$ resembles $C$ more closely than $C^{\epsilon}$ and $C^{\diamond}$ do.

It is interesting to investigate how the relativistic notion of timestamped common knowledge relates to the notions of common knowledge, $\epsilon$-common knowledge, and $\diamond$ common knowledge. Not surprisingly, the relative behavior of the clocks in the system plays a crucial role in determining the meaning of $C^{T}$.

Theorem 12: For any fact $\varphi$ and view-based interpretation,

(a) if it is guaranteed that all clocks show identical times, then at time $T$ on any processor's clock, $C_{G}^{T} \varphi \equiv C_{G} \varphi$.

(b) if it is guaranteed that all clocks are within $\epsilon$ time units of each other, then at time $T$ on any processor's clock, $C_{G}^{T} \varphi \supset C_{G}^{\epsilon} \varphi$.

(c) if it is guaranteed that each local clock reads $T$ at some time, then $C_{G}^{T} \varphi \supset C_{G}^{\diamond} \varphi$.

Theorem 12 gives conditions under which $C^{T}$ can be replaced by $C, C^{\epsilon}$, and $C^{\diamond}$. A weak converse of Theorem 12 holds as well. Suppose the processors are able to set their clocks to a commonly agreed upon time $T$ when they come to know $C_{G} \varphi$ (resp. come to know $\left.C_{G}^{\epsilon} \varphi, C_{G}^{\diamond} \varphi\right)$. Then it is easy to see that whenever $C_{G} \varphi\left(\operatorname{resp} . C_{G}^{\epsilon} \varphi, C_{G}^{\diamond} \varphi\right)$ is attainable, so is $C_{G}^{T} \varphi$.

In many distributed systems timestamped common knowledge seems to be a more appropriate notion to reason about than "true" common knowledge. Although common knowledge cannot be attained in practical systems, timestamped common knowledge is attainable in many cases of interest and seems to correspond closely to the relevant phenomena with which protocol designers are confronted. For example, in distributed protocols that work in phases, we speak of the state of the system at the beginning of 
phase 2, at the end of phase $k$, and so on. It is natural to think of the phase number as a "clock" reading, and consider knowledge about what holds at the different phases as "timestamped" knowledge, with the phase number being the timestamp. In certain protocols for Byzantine agreement, for example, the nonfaulty processors attain common knowledge of the decision value at the end of phase $k$ (cf. [DM90, MT88). In practical systems in which the phases do not end simultaneously at the different sites of the system, the processors can be thought of as actually attaining timestamped common knowledge of the decision value, with the timestamp being "the end of phase $k$ ". Indeed, protocols like the atomic broadcast protocol of [CASD85] are designed exactly for the purpose of attaining timestamped common knowledge. (See $\mathbb{N T 9 3}$ for more discussion of timestamped common knowledge.)

\section{Internal knowledge consistency}

We have seen that common knowledge closely corresponds to the ability to perform simultaneous actions. In the last few sections we introduced a number of related states of knowledge corresponding to weaker forms of coordinated actions. Such weaker forms of coordination are often sufficient for many practical applications. This helps explain the paradox of the happy existence of practical distributed systems despite the apparent need for common knowledge and the negative results of Theorem 8 .

However, there are situations where we act as if - or we would like to carry out our analysis as if - we had true common knowledge, not a weaker variant. For example, in the muddy children puzzle, even though simultaneity may not be attainable, we want to assume that the children do indeed have common knowledge of the father's statement. As another example, consider a protocol that proceeds in phases, in which it is guaranteed that no processor will ever receive a message out of phase. In many cases, all the aspects of this protocol that we may be interested in are faithfully represented if we model the system as if it were truly synchronous: all processors switch from one phase to the next simultaneously.

Intuitively, in both cases, the assumption of common knowledge seems to be a safe one, even if it is not quite true. We would like to make this intuition precise. Recall that an epistemic interpretation is one that specifies what a processor believes at any given point as a function of the processor's history at that point. An epistemic interpretation $\mathcal{I}$ is a knowledge interpretation if it is knowledge consistent, i.e., if it has the property that whenever $(\mathcal{I}, r, t) \models K_{i} \varphi$ then also $(\mathcal{I}, r, t) \models \varphi$. Now an epistemic interpretation that is not knowledge consistent may nevertheless be internally knowledge consistent, which intuitively means that the processors never obtain information from within the system that would contradict the assumption that the epistemic interpretation is in fact a knowledge interpretation. In other words, no processor ever has information that implies that the knowledge axiom $K_{i} \varphi \supset \varphi$ is violated. More formally, an epistemic interpretation $\mathcal{I}$ for a system $R$ is said to be internally knowledge consistent if there is 
a subsystem $R^{\prime} \subseteq R$ such that $\mathcal{I}$ is a knowledge interpretation when restricted to $R^{\prime}$, and for all processors $p_{i}$ and points $(r, t)$ of $R$, there is a point $\left(r^{\prime}, t^{\prime}\right)$ in $R^{\prime}$ such that $h\left(p_{i}, r, t\right)=h\left(p_{i}, r^{\prime}, t^{\prime}\right)$.

Given that epistemic interpretations ascribe knowledge (or, perhaps more appropriately in this case, beliefs) to processors as a function of the processors' histories, the above definition implies that whenever a processor is ascribed knowledge of a certain fact at a point of $R$, then as far as any events involving this processor at the current and at any future time are concerned, it is consistent to assume that the fact does indeed hold.

Using the notion of internal knowledge consistency, we can make our previous intuitions precise. When analyzing the muddy children puzzle, we assume that the children will never discover that they did not hear and comprehend the father's statement simultaneously. We take the set $R^{\prime}$ from the definition of internal knowledge consistency here to be precisely the set of runs where they did hear and comprehend the father's statement simultaneously. Similarly, in the case of the protocol discussed above, the set $R^{\prime}$ is the set where all processors advance from one phase to the next truly simultaneously. It now also makes sense to say that under reasonable conditions processors can safely use an "eager" protocol corresponding to the eager epistemic interpretation of Section 8, in which processors act as if they had common knowledge, even though common knowledge does not hold. It is possible to give a number of conditions on the ordering of events in the system that will ensure that it will be internally knowledge consistent for the processors to act as if they have common knowledge.

For further discussion on internal knowledge consistency, see the recent paper by Neiger $\mathbb{N e i 8 8 .}$.

\section{Conclusions}

In this paper, we have tried to bring out the important role of reasoning about knowledge in distributed systems. We have shown that reasoning about the knowledge of a group and its evolution can reveal subtleties that may not otherwise be apparent, can sharpen our understanding of basic issues, and can improve the high-level reasoning required in the design and analysis of distributed protocols and plans.

We introduced a number of states of group knowledge, but focused much of our attention on one particular state, that of common knowledge. We showed that, in a precise sense, common knowledge is a prerequisite for agreement. However, we also showed that in many practical systems common knowledge is not attainable. This led us to consider three variants of common knowledge - $\epsilon$-common knowledge, eventual common knowledge, and timestamped common knowledge - that are attainable in practice, and may suffice for carrying out a number of actions. The methodology we introduce for constructing these variants of common knowledge, involving the fixed-point operator, can be used to construct other useful variants of common knowledge. Indeed, recent papers 
have introduced concurrent common knowledge [PT92, probabilistic common knowledge [FH94], and polynomial time common knowledge [Mos88], using this methodology.

There is clearly much more work to be done in terms of gaining a better understanding of knowledge in distributed systems. This paper considers a general model of a distributed system. It would also be useful to consider knowledge in distributed systems with particular properties. The work of Chandy and Misra [CM86] is an interesting study of this kind (see [DM90, FHV92, Had87 for other examples). We carried out a knowledge-based analysis of the coordinated attack problem here. Since this paper first appeared, a number of other problems, including Byzantine agreement, distributed commitment, and mutual exclusion, have been analyzed in terms of knowledge (see CM86, DM90, Had87, HZ92, ML90, MT88, NT93|). Such knowledge-based analyses both shed light on the problem being studied and improve our understanding of the methodology. More studies of this kind would further deepen our understanding of the issues involved.

Another general direction of research is that of using knowledge for the specification and verification of distributed systems. (See [KT86] for an initial step in this direction.) Formalisms based on knowledge may prove to be a powerful tool for specifying and verifying protocols, and may also be readily applicable to the synthesis of protocols and plans. Temporal logic has already proved somewhat successful in this regard [EC82, MW84.

Our analysis of the muddy children puzzle and the coordinated attack problem, as well as the work in MDH86, HF85, DM90, MT88 illustrate how subtle the relationship between knowledge, action, and communication in a distributed system can be. In this context, Halpern and Fagin (cf. HF85) look at knowledge-based protocols, which are protocols in which a processor's actions are explicitly based on the processor's knowledge. This provides an interesting generalization of the more standard notions of protocols.

In the long run, we hope that a theory of knowledge, communication, and action will prove rich enough to provide general foundations for a unified theoretical treatment of distributed systems. Such a theory also promises to shed light on aspects of knowledge that are relevant to related fields.

Acknowledgements: This work evolved from work the authors did with Danny Dolev on [MDH86]. Many people commented on different versions of this work. Of special value were comments by Dave Chelberg, Steve Deering, Cynthia Dwork, Ron Fagin, Vassos Hadzilacos, Danny Lehmann, Yoni Malachi, Tim Mann, Andres Modet, Gil Neiger, Jan Pachl, Derek Proudian, Stan Rosenschein, Yoav Shoham, Ray Strong, Moshe Vardi, Joe Weening, and Lenore Zuck. Jan Pachl suggested the term "distributed knowledge", to replace the term "implicit knowledge" that we had been using. We would particularly like to thank Gil Neiger and Lenore Zuck for an outstanding job of refereeing, well beyond the call of duty. 


\section{Appendix A}

In this appendix we present a logic with a greatest fixed point operator and illustrate how common knowledge and variants of common knowledge can be formally defined as greatest fixed points. Our presentation follows that of Kozen KKoz83.

Intuitively, given a system $R$, a formula $\psi$ partitions the points of $R$ into two sets: those that satisfy $\psi$, and those that do not. We can identify a formula with the set of points that satisfy it. In order to be able to define fixed points of certain formulas, which is our objective in this appendix, we consider formulas that may contain a free variable whose values range over subsets of the points of $R$. Once we assign a set of points to the free variable, the formula can be associated with a set of points in a straightforward way (as will be shown below). Thus, such a formula can be viewed as a function from subsets of $R$ to subsets of $R$. (A formula with no free variable is then considered a constant function, yielding the same subset regardless of the assignment.)

Before we define the logic more formally, we need to review a number of relevant facts about fixed points. Suppose $S$ is a set and $f$ is a function mapping subsets of $S$ to subsets of $S$. A subset $A$ of $S$ is said to be a fixed point of $f$ if $f(A)=A$. A greatest (respectively, least) fixed point of $f$ is a set $B$ such that $f(B)=B$, and if $f(A)=A$, then $A \subseteq B$ (resp. $B \subseteq A$ ). It follows that if $f$ has a greatest fixed point $B$, then $B=\bigcup\{A: f(A)=A\}$. The function $f$ is said to be monotone increasing if $f(A) \subseteq f(B)$ whenever $A \subseteq B$ and monotone decreasing if $f(A) \supseteq f(B)$ whenever $A \subseteq B$. The Knaster-Tarski theorem (cf. [Tar55]) implies that a monotone increasing function has a greatest (and a least) fixed point. Given a function $f$ and a subset $A$, define $f^{0}(A)=A$ and $f^{i+1}(A)=f\left(f^{i}(A)\right)$. $f$ is said to be downward continuous if $f\left(\bigcap_{i} A_{i}\right)=\bigcap_{i} f\left(A_{i}\right)$ for all sequences $A_{1}, A_{2}, \ldots$ with $A_{1} \supseteq A_{2} \supseteq$... Given a monotone increasing and downward continuous function $f$ it is not hard to show that the greatest fixed point of $f$ is the set $\bigcap_{k<\omega} f^{k}(S)$. We remark that if $f$ is monotone increasing but not downward continuous, then we can still obtain the greatest fixed points of $f$ in this fashion, but we have to extend the construction by defining $f^{\alpha}$ for all ordinals $\alpha . \emptyset$

We are now in a position to formally define our logic. We start with a set $\Phi=$ $\left\{P, Q, P_{1}, \ldots\right\}$ of primitive propositions and a single propositional variable $X$. We form more complicated formulas by allowing the special formula true and then closing off under conjunction, negation, the modal operators $K_{i}, E_{G}, E_{G}^{\epsilon}$, and $E_{G}^{\diamond}$ for every group $G$ of processors, and the greatest fixed point operator $\nu X$. Thus, if $\varphi$ and $\psi$ are formulas, then so are $\neg \varphi, \varphi \wedge \psi, K_{i} \varphi, E_{G} \varphi, E_{G}^{\epsilon} \varphi, E_{G}^{\diamond} \varphi$, and $\nu X . \varphi$ (read "the greatest fixed point of $\varphi$ with respect to $\left.X^{\prime \prime}\right)$. However, we place a syntactic restriction, described below, on formulas of the form $\nu X . \varphi$.

Just as $\forall x$ in first-order logic binds occurrences of $x, \nu X$ binds occurrences of $X$.

\footnotetext{
${ }^{8}$ We can similarly define a function $f$ to be upward continuous if $f\left(\bigcup_{i} A_{i}\right)=\bigcup_{i} f\left(A_{i}\right)$ for all sequences $A_{1}, A_{2}, \ldots$ with $A_{1} \subseteq A_{2} \subseteq \ldots$. For monotone increasing upward continuous functions $f$, the least fixed point of $f$ is $\bigcup_{k<\omega} f^{k}(\emptyset)$. Again, to get least fixed points in the general case, we have to extend this construction through the ordinals.
} 


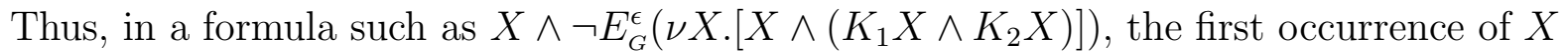
is free, while the rest are bound. We say that a free occurrence of $X$ in a formula $\varphi$ is positive if it is in the scope of an even number of negation signs, and negative if it is in the scope of an odd number of negation signs. Thus, in a formula such as $X \wedge \neg K_{1} X$, the first occurrence of $X$ is positive while the second is negative. The restriction on formulas of the form $\nu X . \varphi$ is that all free occurrences of $X$ in $\varphi$ must be positive; the point of this restriction will be explained below.

The next step is to associate with each formula a function. Given a distributed system represented by its set of runs $R$, let $S=R \times[0, \infty)$. A model $\mathcal{M}$ is a triple $(S, \pi, v)$, where $S$ is as above, $\pi$ associates a truth assignment to the primitive propositions with each point in $S$, and $v:\{1, \ldots, m\} \times S \rightarrow \Sigma$ is an assignment of views (from a set of states $\Sigma$ ) to the processors at the points of $S$. We now associate with each formula $\varphi$ a function $\varphi^{\mathcal{M}}$ from subsets of $S$ to subsets of $S$. Intuitively, if no occurrences of $X$ are free in $\varphi$, then $\varphi^{\mathcal{M}}$ will be a constant function, and $\varphi^{\mathcal{M}}(A)$ will be the set of points where $\varphi$ is true (no matter how we choose $A$ ). If $X$ is free in $\varphi$, then $\varphi^{\mathcal{M}}(A)$ is the set of points where $\varphi$ is true if $A$ is the set of points where $X$ is true. We define $\varphi^{\mathcal{M}}(A)$ by induction on the structure of $\varphi$ as follows:

(a) $X^{\mathcal{M}}(A)=A$ (so $X^{\mathcal{M}}$ is the identity function).

(b) $P^{\mathcal{M}}(A)=\{s \in S: \pi(s)(P)=$ true $\}$ for a primitive proposition $P$

(c) $\operatorname{true}^{\mathcal{M}}(A)=S$.

(d) $(\neg \varphi)^{\mathcal{M}}(A)=S-\varphi^{\mathcal{M}}(A)$.

(e) $(\varphi \wedge \psi)^{\mathcal{M}}(A)=\varphi^{\mathcal{M}}(A) \cap \psi^{\mathcal{M}}(A)$.

(f) $\left(K_{i} \varphi\right)^{\mathcal{M}}(A)=\left\{(r, t) \in S\right.$ : for all $\left(r^{\prime}, t^{\prime}\right) \in S, v\left(p_{i}, r, t\right)=v\left(p_{i}, r^{\prime}, t^{\prime}\right)$ implies $\left.\left(r^{\prime}, t^{\prime}\right) \in \varphi^{\mathcal{M}}(A)\right\}$.

(g) $\left(E_{G} \varphi\right)^{\mathcal{M}}(A)=\cap_{i \in G}\left(K_{i} \varphi\right)^{\mathcal{M}}(A)$.

(h) $\left(E_{G}^{\epsilon} \varphi\right)^{\mathcal{M}}(A)=\left\{(r, t) \in S\right.$ : there exists an interval $I=\left[t^{\prime}, t^{\prime}+\epsilon\right]$ with $t \in I$, such that $\left.\forall p_{i} \in G \exists t_{i} \in I\left(\left(r, t_{i}\right) \in\left(K_{i} \varphi\right)^{\mathcal{M}}(A)\right)\right\}$.

(i) $\left(E_{G}^{\diamond} \varphi\right)^{\mathcal{M}}(A)=\left\{(r, t) \in S: \forall p_{i} \in G \exists t_{i}\left(\left(r, t_{i}\right) \in\left(K_{i} \varphi\right)^{\mathcal{M}}(A)\right)\right\}$.

(j) $(\nu X . \varphi)^{\mathcal{M}}(A)=\bigcup\left\{B: \varphi^{\mathcal{M}}(B)=B\right\}$.

Now by an easy induction on the structure of formulas we can prove the following facts:

1. If $\varphi$ is a formula in which all free occurrences of $X$ are positive (resp. negative), then $\varphi^{\mathcal{M}}$ is monotone increasing (resp. monotone decreasing). Note that our syntactic restriction on formulas of the form $\nu X . \varphi$ guarantees that for a well-formed formula of this form, the function $\varphi^{\mathcal{M}}$ is monotone increasing. As a consequence, $(\nu X . \varphi)^{\mathcal{M}}(A)$ is the greatest fixed point of the function $\varphi^{\mathcal{M}}$. 
2. If $\varphi$ is a formula with no free variables, then $\varphi^{\mathcal{M}}$ is a constant function. In particular, observe that $(\nu X . \varphi)^{\mathcal{M}}$ is necessarily a constant function (the definition shows that $(\nu X . \varphi)^{\mathcal{M}}(A)$ is independent of the choice of $\left.A\right)$. As well, it is easy to check that if $\varphi$ is a valid formula such as $\neg(P \wedge \neg P)$, then $\varphi^{\mathcal{M}}(A)=S$.

3. For formulas in which the variable $X$ does not appear (so, in particular, for formulas not involving the greatest fixed point operator), $\varphi^{\mathcal{M}}(A)=\left\{(r, t):\left(\mathcal{I}_{v}, r, t\right) \models \varphi\right\}$, where $\mathcal{I}_{v}$ is the view-based interpretation associated with the view function $v$. (Again, this is true for any choice of $A$, since by the previous observation, $\varphi^{\mathcal{M}}$ is a constant function if there is no occurrence of $X$ in $\varphi$.) Thus, if we define $(\mathcal{M}, r, t) \models \varphi$ iff $(r, t) \in \varphi^{\mathcal{M}}(\emptyset)$, then this definition extends our previous definition (in that for formulas in which the variable $X$ does not appear, we have $(\mathcal{M}, r, t) \models \varphi$ iff $\left.\left(\mathcal{I}_{v}, r, t\right) \models \varphi\right)$.

Given the machinery at our disposal, we can now formally define $C_{G} \varphi$ as $\nu X . E_{G}(\varphi \wedge$ $X)$, define $C_{G}^{\epsilon} \varphi$ as $\nu X . E_{G}^{\epsilon}(\varphi \wedge X)$, and define $C_{G}^{\diamond} \varphi$ as $\nu X . E_{G}^{\diamond}(\varphi \wedge X)$. It follows from our characterization of greatest fixed points of downward continuous functions that if $\varphi^{\mathcal{M}}$ is downward continuous, then $\nu X . \varphi$ is equivalent to $\varphi_{0} \wedge \varphi_{1} \wedge \ldots$, where $\varphi_{0}$ is true, $\varphi_{i+1}$ is $\varphi\left[\varphi_{i} / X\right]$, and $\varphi[\psi / X]$ denotes the result of substituting $\psi$ for the free occurrences of $X$ in $\varphi$. It is easy to check that $\left(E_{G}(\varphi \wedge X)\right)^{\mathcal{M}}$ is downward continuous if $\varphi^{\mathcal{M}}$ is downward continuous. In particular, if $\varphi$ has no free occurrences of $X$ (so that $\varphi^{\mathcal{M}}$ is constant), it follows that we have:

$$
C_{G} \varphi \equiv E_{G} \varphi \wedge E_{G}\left(\varphi \wedge E_{G} \varphi\right) \wedge E_{G}\left(\varphi \wedge E_{G}\left(\varphi \wedge E_{G} \varphi\right)\right) \wedge \ldots \text { ? }
$$

Since $E_{G}\left(\psi_{1} \wedge \psi_{2}\right) \equiv\left(E_{G} \psi_{1} \wedge E_{G} \psi_{2}\right)$ it follows that

$$
C_{G} \varphi \equiv E_{G} \varphi \wedge E_{G} E_{G} \varphi \wedge \cdots
$$

However, $\left(E_{G}^{\epsilon}(\varphi \wedge X)\right)^{\mathcal{M}}$ and $\left(E_{G}^{\diamond}(\varphi \wedge X)\right)^{\mathcal{M}}$ are not necessarily downward continuous. The reason that $\left(E_{G}^{\diamond}(\varphi \wedge X)\right)^{\mathcal{M}}$ is not downwards continuous is that an infinite collection of facts can each eventually hold, without them necessarily all holding simultaneously at some point. We have already seen one example of this phenomenon in Section 11. For another example, suppose we are working in a system with an unbounded global clock, and let $A_{i}=(\text { current_time }>i)^{\mathcal{M}}$. Since the clock is unbounded, it follows that $A_{i} \neq \emptyset$ for all $i$, but $\cap_{i} A_{i}=\emptyset$. Taking $\psi$ to be the formula $E^{\diamond}(\varphi \wedge X)$, it is easy to see that $(r, 0) \in \psi^{\mathcal{M}}\left(A_{i}\right)$ for all $i$, and hence $\cap_{i}\left(\psi^{\mathcal{M}}\left(A_{i}\right)\right) \neq \psi^{\mathcal{M}}\left(\cap_{i} A_{i}\right)$.

We can construct a similar example in the case of $E^{\epsilon}$, because we have taken time to range over the reals. For example, if we take $x_{i}$ to be an infinite sequence of real numbers coverging from below to $\epsilon$, take $A_{i}=\left(\text { current_time } \in\left(x_{i}, \epsilon\right)\right)^{\mathcal{M}}$, and now take $\psi$ to be

\footnotetext{
${ }^{9}$ Note that the formula on the right-hand side of the equivalence is not in our language, since we have not allowed infinite conjunctions. However, we can easily extend the language to allow infinite conjunctions in the obvious way so that the equivalence holds.
} 
the formula $E^{\epsilon}(\varphi \wedge X)$, then again we have $\cap_{i} A_{i}=\emptyset$, and $(r, 0) \in \cap_{i}\left(\psi^{\mathcal{M}}\left(A_{i}\right)\right)$. This example does depend crucially on the fact that time ranges over the reals. If instead we had taken time to range over the natural numbers, then would in fact get downward continuity.

We encourage the reader to check that $C_{G} \varphi, C_{G}^{\epsilon} \varphi$, and $C_{G}^{\diamond} \varphi$ all satisfy the fixed point axiom and the induction rule. The fixed point axiom is a special case of the more general fixed point axiom $\nu X . \varphi \equiv \varphi[\nu X . \varphi / X]$, while the induction rule is a special case of the more general induction rule for fixed points: from $\psi \supset \varphi[\psi / X]$ infer $\psi \supset \nu X$. $\varphi$. The reader might also now wish to check that $C$ has the properties of S5, while $C^{\epsilon}$ and $C^{\diamond}$ satisfy the positive introspection axiom and the necessitation rule. Furthermore, for stable fact $\varphi$ and complete-history interpretations, they also satisfy the consequence closure axiom. $C^{\epsilon}$ and $C^{\diamond}$ satisfy neither the knowledge axiom nor the negative introspection axiom. We remark that both notions satisfy weaker variants of the knowledge axiom: $C^{\epsilon} \varphi$ implies that $\varphi$ holds at some point at most $\epsilon$ time units away from the current point, while $C^{\diamond} \varphi$ implies that $\varphi$ holds (at least) at some point during the run.

It is straightforward to extend the above framework to include explicit individual clock times in order to define $C_{G}^{T} \varphi$ (see $\mathbb{N T 9 3}$ for more details). Here, for example, it is the case that $\left(E^{T}(\varphi \wedge X)\right)^{\mathcal{M}}$ is downward continuous, and $E^{T}$ distributes over conjunction; hence $C^{T}$ will coincide with the appropriate infinite conjunction. Similar treatments can be applied to many related variants of common knowledge (see, for example, [FH94, Mos88, PT92]).

\section{Appendix B}

In this appendix we fill in the details of the proof that common knowledge cannot be attained in practical systems (Theorem 8 in Section 8).

Our first step is to establish a general condition - namely, that the initial point of a run is reachable from any later point - under which common knowledge can be neither gained nor lost. We remark that Chandy and Misra have shown that in the case of completely asynchronous, event-driven systems where communication is not guaranteed, common knowledge of any fact can be neither gained nor lost [CM86]. Since it is easy to see that, in such systems, the initial point of a run is reachable from all later points, our result provides a generalization of that of [CM86].

Proposition 13: Let $r \in R$ be a run in which the point $(r, 0)$ is $G$-reachable from $(r, t)$ in the graph corresponding to the complete-history interpretation, and let $\mathcal{I}$ be a knowledge interpretation for $R$. Then for all formulas $\varphi$ we have $(\mathcal{I}, r, t) \models C_{G} \varphi$ iff $(\mathcal{I}, r, 0) \models C_{G} \varphi$.

Proof: Fix a run $r$, time $t$, and formula $\varphi$. Since $(r, 0)$ is $G$-reachable from $(r, t)$ in the graph corresponding to the complete-history interpretation, there exist points $\left(r_{0}, t_{0}\right)$, $\left(r_{1}, t_{1}\right), \ldots,\left(r_{k}, t_{k}\right)$ such that $(r, t)=\left(r_{0}, t_{0}\right),(r, 0)=\left(r_{k}, t_{k}\right)$, and for every $i<k$ there 
is a processor $j_{i} \in G$ that has the same history at $\left(r_{i}, t_{i}\right)$ and at $\left(r_{i+1}, t_{i+1}\right)$. We can now prove by induction on $i$, using Lemma 2, that $(\mathcal{I}, r, t) \models C_{G} \varphi$ iff $\left(\mathcal{I}, r_{i}, t_{i}\right) \models C_{G} \varphi$. The result follows.

We next provide a formal definition of systems with temporal imprecision, and show that in such systems, the initial point of a run is always reachable from later points. A system $R$ has temporal imprecision if

$$
\begin{aligned}
& \forall r \in R \forall t \geq 0 \forall i \forall j \neq i \exists \delta>0 \forall \delta^{\prime} \in[0, \delta) \exists r^{\prime} \forall t^{\prime}<t \\
& \left.\quad\left(h\left(p_{i}, r, t^{\prime}\right)=h\left(p_{i}, r^{\prime}, t^{\prime}+\delta^{\prime}\right) \wedge h\left(p_{j}, r, t^{\prime}\right)=h\left(p_{j}, r^{\prime}, t^{\prime}\right)\right)\right) .
\end{aligned}
$$

Intuitively, this means that processors cannot perfectly coordinate their notions of time in a system with temporal imprecision. One processor might always be a little behind the others.

By reachable in the following lemma we mean reachable (in the sense of Section 6) with respect to the view function defined by the complete-history interpretation.

Lemma 14: If $R$ is a system with temporal imprecision, then for all runs $r \in R$ and times $t$, the point $(r, 0)$ is reachable from $(r, t)$.

Proof: $\quad$ Let $R$ be a system with temporal imprecision and $(r, t)$ be a point of $R$. Suppose $t \neq 0$ (otherwise clearly $(r, 0)$ is reachable from $(r, t)$ ). Let $t_{0}$ be the greatest lower bound of the set $\left\{t^{\prime}:\left(r, t^{\prime \prime}\right)\right.$ is reachable from $(r, t)$ for all $\left.t^{\prime \prime} \in\left[t^{\prime}, t\right]\right\}$. We will show that $\left(r, t_{0}\right)$ is reachable from $(r, t)$ and that $t_{0}=0$. Since $R$ is a system with temporal imprecision, there exists a $\delta$ such that for all $\delta^{\prime}$ with $0<\delta^{\prime}<\delta$, there exists a run $r^{\prime}$ such that for all $t^{\prime} \leq t$, we have $h\left(p_{1}, r, t^{\prime}\right)=h\left(p_{1}, r^{\prime}, t^{\prime}+\delta^{\prime}\right)$ and $h\left(p_{i}, r, t^{\prime}\right)=h\left(p_{i}, r^{\prime}, t^{\prime}\right)$ for $i \neq 1$. If $\delta^{\prime}<t^{\prime} \leq t$, it follows that $\left(r^{\prime}, t^{\prime}\right)$ is reachable from $\left(r, t^{\prime}\right)$ and $\left(r, t^{\prime}-\delta^{\prime}\right)$ is reachable from $\left(r^{\prime}, t^{\prime}\right)$. By transitivity of reachability, we have that $\left(r, t^{\prime}-\delta^{\prime}\right)$ is reachable from $\left(r, t^{\prime}\right)$, and by symmetry, that $\left(r, t^{\prime}\right)$ is reachable from $\left(r, t^{\prime}-\delta^{\prime}\right)$. It now follows that $\left(r, t-\delta^{\prime}\right)$ is reachable from $(r, t)$ for all $\delta^{\prime}<\min (\delta, t)$. Thus $t_{0} \leq t-\min (\delta, t)$. Furthermore, if $\delta^{\prime}<\min (\delta, t)$, then we know that $\left(r, t_{0}+\delta^{\prime}\right)$ is reachable from both $\left(r, t_{0}\right)$ and $(r, t)$. It thus follows that $\left(r, t_{0}\right)$ is reachable from $(r, t)$. Finally, if $t_{0} \neq 0$, then we know that $\left(r, t_{0}-\delta^{\prime}\right)$ is reachable from $\left(r, t_{0}\right)$ (and hence from $\left.(r, t)\right)$ for all $\delta^{\prime}<\min \left(t_{0}, \delta\right)$. But this contradicts our choice of $t_{0}$. Thus $t_{0}=0$, and $(r, 0)$ is reachable from $(r, t)$.

Theorem 8 now follows as an immediate corollary to Lemma 14 and Proposition 13.

We conclude by showing that many practical systems do indeed have temporal imprecision (although the $\delta$ 's involved in some cases might be very small). Perhaps through statistical data, we can assume that for every communication link $l$ there are known lower and upper bounds $L_{l}$ and $H_{l}$ respectively on the message delivery time for messages over $l$. We assume that the message delivery time on the link $l$ is always in the open interval $\left(L_{l}, H_{l}\right)$. (We take the interval to be open here since it seems reasonable to suppose that if the system designer considers it possible that a message will take time $T$ to be delivered, then for some sufficiently small $\delta>0$, he will also consider it possible that 
the delivery time is anywhere in the interval $(T-\delta, T+\delta)$; in this we differ slightly from [DHS86, HMM85].) We define $f_{l}$ to be a message delivery function for link $l$ if $f_{l}: \mathbf{N} \rightarrow\left(L_{l}, H_{l}\right)$. A run $r$ is consistent with $f_{l}$ if for all $n \in \mathbf{N}, f_{l}(n)$ is the delivery time of the $n^{\text {th }}$ message in $r$ on link $l$. A system $R$ has bounded but uncertain message delivery times if for all links $l$ there exist bounds $L_{l}<H_{l}$ such that for all runs $r \in R$ and all message delivery functions $f_{l}: \mathbf{N} \rightarrow\left(L_{l}, H_{l}\right)$, there exists a run $r^{\prime}$ which is identical to $r$ except that message delivery time over the link $l$ is defined by $f_{l}$. More formally, $r^{\prime}$ is consistent with $f_{l}$ and for all $i$, processor $p_{i}$ follows the same protocol, wakes up at the same time (i.e., $t_{\text {init }}\left(p_{i}, r\right)=t_{\text {init }}\left(p_{i}, r^{\prime}\right)$ ), and has the same initial state and the same clock readings in both $r$ and $r^{\prime}$.

We say $R$ is a system with uncertain start times if there exists $\delta_{0}>0$ such that given a run $r \in R$, a processor $p_{i}$, and $\delta$ with $0<\delta<\delta_{0}$, there is a run $r^{\prime}$ which is identical to $r$ except that $p_{i}$ wakes up $\delta$ earlier in $r^{\prime}$ with its clock readings (if there are clocks in the system) shifted back by $\delta$. More formally, for all $j \neq i$, processor $p_{j}$ follows the same protocol, wakes up at the same time, and has the same initial state in both $r$ and $r^{\prime}$. Moreover, for all $k$, the delivery time for the $k^{\text {th }}$ message on link $l$ (if there is one) is the same in both $r$ and $r^{\prime}$. All processors other than $p_{i}$ have the same clock readings in both $r$ and $r^{\prime}$. Processor $p_{i}$ starts $\delta$ later in $r^{\prime}$ than $r$, although it has the same initial state in both runs, and $\tau\left(p_{i}, r, t\right)=\tau\left(p_{i}, r^{\prime}, t+\delta\right)$.

For any practical system, it seems reasonable to assume that there will be some (perhaps very small) uncertainty in start times and, even if message delivery is guaranteed within a bounded time, that there is some uncertainty in message delivery time. These assumptions are sufficient to guarantee temporal imprecision, as the following result, whose proof is a slight modification of a result proved in [DHS86 on the tightness of clock synchronization achievable, shows:

Proposition 15: A system with bounded but uncertain message delivery times and uncertain start times has temporal imprecision.

Sketch of Proof: Let $(r, t)$ be a point of the system, and let $p_{i}$ be a processor. Let $\delta_{0}$ be as in the definition of uncertain start times. Since only a finite number of messages are received by time $t$ in $r$, there is some $\delta>0$ such that the delivery times of these messages are more than $\delta$ greater than the lower bound for the particular link they were sent over, and more than $\delta$ less than the upper bound. Choose $\delta^{\prime}<\min \left(\delta_{0}, \delta\right)$ and some processor $p_{i}$. Let $r^{\prime}$ be a run in which all processors $p_{j} \neq p_{i}$ start at the same time and in the same initial state as in $r$, have the same clock readings (if there are clocks), and all messages between such processors take exactly the same time as in $r$. In addition, processor $p_{i}$ starts $\delta^{\prime}$ time units later in $r^{\prime}$ than in $r$, messages to $p_{i}$ take $\delta^{\prime}$ time units longer to be delivered, while messages from $p_{i}$ are delivered $\delta^{\prime}$ time units faster than in $r$, and $p_{i}$ 's clock readings (if there are clocks) are shifted by $\delta^{\prime}$. Such a run $r^{\prime}$ exists by our assumptions. It is not hard to check that run $r^{\prime}$ has the property that for all times $t^{\prime} \leq t$, all processors $p_{j} \neq p_{i}$ have exactly the same history at time $t^{\prime}$ in both $r$ and $r^{\prime}$, while 
processor $p_{i}$ has the same history at $\left(r, t^{\prime}\right)$ and at $\left(r^{\prime}, t^{\prime}+\delta^{\prime}\right)$. Since $(r, t)$ and $p_{i}$ were chosen arbitrarily, it thus follows that the system has temporal imprecision.

\section{References}

[Aum76] R. J. Aumann. Agreeing to disagree. Annals of Statistics, 4(6):1236-1239, 1976.

[Bar81] J. Barwise. Scenes and other situations. Journal of Philosophy, 78(7):369-397, 1981.

[CASD85] F. Cristian, H. Aghili, H. R. Strong, and D. Dolev. Atomic broadcast: from simple diffusion to Byzantine agreement. In Proc. 15th International Conf. on Fault-Tolerant Computing Systems, 1985.

[CL85] K. M. Chandy and L. Lamport. Distributed snapshots: determining global states of distributed systems. ACM Trans. on Computer Systems, 3(1):63-75, 1985 .

[CM81] H. H. Clark and C. R. Marshall. Definite reference and mutual knowledge. In A. K. Joshi, B. L. Webber, and I. A. Sag, editors, Elements of discourse understanding. Cambridge University Press, Cambridge, U.K., 1981.

[CM86] K. M. Chandy and J. Misra. How processes learn. Distributed Computing, 1(1):40-52, 1986.

[DHS86] D. Dolev, J. Y. Halpern, and H. R. Strong. On the possibility and impossibility of achieving clock synchronization. Journal of Computer and System Sciences, $32(2): 230-250,1986$.

[DM90] C. Dwork and Y. Moses. Knowledge and common knowledge in a Byzantine environment: crash failures. Information and Computation, 88(2):156-186, 1990.

[DRS90] D. Dolev, R. Reischuk, and H. R. Strong. Early stopping in Byzantine agreement. Journal of the ACM, 34(7):720-741, 1990.

[EC82] E. A. Emerson and E. M. Clarke. Using branching time temporal logic to synthesize synchronization skeletons. Science of Computer Programming, 2:241$266,1982$.

[FH88] R. Fagin and J. Y. Halpern. Belief, awareness, and limited reasoning. Artificial Intelligence, 34:39-76, 1988. 
[FH94] R. Fagin and J. Y. Halpern. Reasoning about knowledge and probability. Journal of the ACM, 41(2):340-367, 1994.

[FHV92] R. Fagin, J. Y. Halpern, and M. Y. Vardi. What can machines know? On the properties of knowledge in distributed systems. Journal of the ACM, 39(2):328-376, 1992.

[FI86] M. J. Fischer and N. Immerman. Foundations of knowledge for distributed systems. In J. Y. Halpern, editor, Theoretical Aspects of Reasoning about Knowledge: Proc. 1986 Conference, pages 171-186. Morgan Kaufmann, San Francisco, Calif., 1986.

[FLP85] M. J. Fischer, N. A. Lynch, and M. S. Paterson. Impossibility of distributed consensus with one faulty processor. Journal of the ACM, 32(2):374-382, 1985.

[Gal79] R. G. Gallager. Seminar on computer communication networks, Office of Industrial Liason, MIT. 1979.

[Gra78] J. Gray. Notes on database operating systems. In R. Bayer, R. M. Graham, and G. Seegmuller, editors, Operating Systems: An Advanced Course, Lecture Notes in Computer Science, Vol. 66. Springer-Verlag, Berlin/New York, 1978. Also appears as IBM Research Report RJ 2188, 1978.

[Had87] V. Hadzilacos. A knowledge-theoretic analysis of atomic commitment protocols. In Proc. 6th ACM Symp. on Principles of Database Systems, pages 129-134, 1987. A revised version has been submitted for publication.

[Hal87] J. Y. Halpern. Using reasoning about knowledge to analyze distributed systems. In J. F. Traub, B. J. Grosz, B. W. Lampson, and N. J. Nilsson, editors, Annual Review of Computer Science, Vol. 2, pages 37-68. Annual Reviews Inc., Palo Alto, Calif., 1987.

[HF85] J. Y. Halpern and R. Fagin. A formal model of knowledge, action, and communication in distributed systems: preliminary report. In Proc. 4th ACM Symp. on Principles of Distributed Computing, pages 224-236, 1985.

[Hin62] J. Hintikka. Knowledge and Belief. Cornell University Press, Ithaca, N.Y., 1962.

[HM90] J. Y. Halpern and Y. Moses. Knowledge and common knowledge in a distributed environment. Journal of the ACM, 37(3):549-587, 1990. A preliminary version appeared in Proc. 3rd ACM Symposium on Principles of Distributed Computing, 1984. 
[HM92] J. Y. Halpern and Y. Moses. A guide to completeness and complexity for modal logics of knowledge and belief. Artificial Intelligence, 54:319-379, 1992.

[HMM85] J. Y. Halpern, N. Megiddo, and A. Munshi. Optimal precision in the presence of uncertainty. Journal of Complexity, 1:170-196, 1985.

[HZ92] J. Y. Halpern and L. D. Zuck. A little knowledge goes a long way: knowledgebased derivations and correctness proofs for a family of protocols. Journal of the ACM, 39(3):449-478, 1992.

[Koz83] D. Kozen. Results on the propositional $\mu$-calculus. Theoretical Computer Science, 27(1):333-354, 1983.

[KT86] S. Katz and G. Taubenfeld. What processes know: definitions and proof methods. In Proc. 5th ACM Symp. on Principles of Distributed Computing, pages 249-262, 1986.

[Lev84] H. J. Levesque. A logic of implicit and explicit belief. In Proc. National Conference on Artificial Intelligence (AAAI '84), pages 198-202, 1984.

[LR86] R. E. Ladner and J. H. Reif. The logic of distributed protocols (preliminary report). In J. Y. Halpern, editor, Theoretical Aspects of Reasoning about Knowledge: Proc. 1986 Conference, pages 207-222. Morgan Kaufmann, San Francisco, Calif., 1986.

[MDH86] Y. Moses, D. Dolev, and J. Y. Halpern. Cheating husbands and other stories: a case study of knowledge, action, and communication. Distributed Computing, 1(3):167-176, 1986.

[ML90] M. S. Mazer and F. H. Lochovsky. Analyzing distributed commitment by reasoning about knowledge. Technical Report CRL 90/10, DEC-CRL, 1990.

[Moo85] R. C. Moore. A formal theory of knowledge and action. In J. Hobbs and R. C. Moore, editors, Formal Theories of the Commonsense World, pages 319-358. Ablex Publishing Corp., Norwood, N.J., 1985.

[Mos88] Y. Moses. Resource-bounded knowledge. In M. Y. Vardi, editor, Proc. Second Conference on Theoretical Aspects of Reasoning about Knowledge, pages 261276. Morgan Kaufmann, San Francisco, Calif., 1988.

[MSHI79] J. McCarthy, M. Sato, T. Hayashi, and S. Igarishi. On the model theory of knowledge. Technical Report STAN-CS-78-657, Stanford University, 1979.

[MT88] Y. Moses and M. R. Tuttle. Programming simultaneous actions using common knowledge. Algorithmica, 3:121-169, 1988. 
[MW84] Z. Manna and P. L. Wolper. Synthesis of communicating processes from temporal logic specifications. ACM Trans. on Programming Languages and Systems, 6(1):68-93, 1984.

[Nei88] G. Neiger. Knowledge consistency: a useful suspension of disbelief. In M. Y. Vardi, editor, Proc. Second Conference on Theoretical Aspects of Reasoning about Knowledge, pages 295-308. Morgan Kaufmann, San Francisco, Calif., 1988.

[NT93] G. Neiger and S. Toueg. Simulating real-time clocks and common knowledge in distributed systems. Journal of the ACM, 40(2):334-367, 1993.

[PR85] R. Parikh and R. Ramanujam. Distributed processing and the logic of knowledge. In R. Parikh, editor, Proc. Workshop on Logics of Programs, pages 256-268, 1985.

[PT92] P. Panangaden and S. Taylor. Concurrent common knowledge: defining agreement for asynchronous systems. Distributed Computing, 6(2):73-93, 1992.

[RK86] S. J. Rosenschein and L. P. Kaelbling. The synthesis of digital machines with provable epistemic properties. In J. Y. Halpern, editor, Theoretical Aspects of Reasoning about Knowledge: Proc. 1986 Conference, pages 83-97. Morgan Kaufmann, San Francisco, Calif., 1986.

[Ros85] S. J. Rosenschein. Formal theories of AI in knowledge and robotics. New Generation Computing, 3:345-357, 1985.

[Tar55] A. Tarski. A lattice-theoretic fixpoint theorem and its applications. Pacific Journal of Mathematics, 5:285-309, 1955.

[YC79] Y. Yemini and D. Cohen. Some issues in distributed processes communication. In Proc. of the 1st International Conf. on Distributed Computing Systems, pages 199-203, 1979. 